\title{
TOA-Based Robust Wireless Geolocation and Cramér-Rao Lower Bound Analysis in Harsh LOS/NLOS Environments
}

\author{
Feng Yin, Carsten Fritsche, Fredrik Gustafsson and Abdelhak M Zoubir
}

\section{Linköping University Post Print}

\section{Tweet}

N.B.: When citing this work, cite the original article.

(C2013 IEEE. Personal use of this material is permitted. However, permission to reprint/republish this material for advertising or promotional purposes or for creating new collective works for resale or redistribution to servers or lists, or to reuse any copyrighted component of this work in other works must be obtained from the IEEE.

Feng Yin, Carsten Fritsche, Fredrik Gustafsson and Abdelhak M Zoubir, TOA-Based Robust Wireless Geolocation and Cramér-Rao Lower Bound Analysis in Harsh LOS/NLOS Environments, 2013, IEEE Transactions on Signal Processing, (61), 9, 2243-2255.

http://dx.doi.org/10.1109/TSP.2013.2251341

Postprint available at: Linköping University Electronic Press

http://urn.kb.se/resolve?urn=urn:nbn:se:liu:diva-92694 


\title{
TOA Based Robust Wireless Geolocation and Cramér-Rao Lower Bound Analysis in Harsh LOS/NLOS Environments
}

\author{
Feng Yin, Student Member, IEEE, Carsten Fritsche, Member, IEEE, Fredrik Gustafsson, Fellow, IEEE \\ and Abdelhak M. Zoubir, Fellow, IEEE
}

\begin{abstract}
We consider time-of-arrival based robust geolocation in harsh line-of-sight/non-line-of-sight environments. Herein, we assume the probability density function (PDF) of the measurement error to be completely unknown and develop an iterative algorithm for robust position estimation. The iterative algorithm alternates between a PDF estimation step, which approximates the exact measurement error PDF (albeit unknown) under the current parameter estimate via adaptive kernel density estimation, and a parameter estimation step, which resolves a position estimate from the approximate log-likelihood function via a quasi-Newton method. Unless the convergence condition is satisfied, the resolved position estimate is then used to refine the PDF estimation in the next iteration. We also present the best achievable geolocation accuracy in terms of the CramérRao lower bound. Various simulations have been conducted in both real-world and simulated scenarios. When the number of received range measurements is large, the new proposed position estimator attains the performance of the maximum likelihood estimator (MLE). When the number of range measurements is small, it deviates from the MLE, but still outperforms several salient robust estimators in terms of geolocation accuracy, which comes at the cost of higher computational complexity.
\end{abstract}

Index Terms-Adaptive kernel density estimation (AKDE), Cramér-Rao lower bound (CRLB), non-line-of-sight (NLOS) mitigation, robust geolocation, time-of-arrival (TOA).

\section{INTRODUCTION}

Wireless geolocation refers to the problem of finding the position of a mobile subscriber in different wireless systems, such as cellular networks, wireless local area networks, and wireless sensor networks [1]. Over the past two decades, wireless geolocation has received considerable attention due to the expanding location-based services, such as wireless Emergency 911 (E-911) service, location-sensitive billing, fraud detection, intelligent transportation systems and mobile yellow pages [2]. In order to give a position estimate, most geolocation systems utilize a two-step geolocation technique

Copyright (C)2012 IEEE. Personal use of this material is permitted. However, permission to use this material for any other purposes must be obtained from the IEEE by sending a request to pubs-permissions@ieee.org.

F. Yin and A. M. Zoubir are with the Signal Processing Group, Institute of Telecommunications, Technische Universität Darmstadt, Darmstadt, 64283, Germany (e-mail: fyin@spg.tu-darmstadt.de; zoubir@spg.tu-darmstadt.de).

C. Fritsche was with the Department of Electrical Engineering, Division of Automatic Control, Linköping University, Linköping, SE-581 83, Sweden. He is now with the IFEN GmbH, Poing, 85586, Germany (e-mail: carsten@isy.liu.se).

F. Gustafsson is with the Department of Electrical Engineering, Division of Automatic Control, Linköping University, Linköping, SE-581 83, Sweden (e-mail: fredrik@isy.liu.se). primarily due to its lower complexity as compared to a direct geolocation technique (see e.g., [3], [4]). In the first step of the two-step geolocation technique, position related measurements such as received-signal-strength (RSS), time-of-arrival (TOA), time-difference-of-arrival (TDOA) and angle-of-arrival (AOA) are extracted. In the second step, the obtained measurements are processed so as to give a position estimate. Various different position estimation approaches can be found in [5][8] and references therein.

In indoor environments or urban areas, non-line-of-sight (NLOS) propagation significantly degrades the estimation performance of conventional position estimation techniques (e.g., [9]-[14]), which are developed solely under the line-of-sight (LOS) assumption. Therefore, robust methods for position estimation are constantly sought after. In this paper, we focus on the design of a new robust position estimation approach based on the extracted TOA measurements. The existing TOA based robust position estimation approaches can be broadly categorized into "identify and discard" based approaches, "mathematical programming" based approaches and robust estimation based approaches.

The essence of the "identify and discard" based approaches (see e.g., [15]-[17]) is to identify and discard those NLOScorrupted range measurements. The remaining range measurements, classified as LOS measurements, are then used by conventional algorithms (e.g., least-squares and maximum likelihood algorithms) to compute an accurate position estimate. The key idea of "mathematical programming" based approaches is to formulate the position estimation problem as a constraint optimization problem, which can be solved by mathematical programming techniques (e.g., quadratic programming [18], linear programming [19], and semi-definite programming [20]). In order to combat the NLOS effect, robust estimation based approaches resort to replace the leastsquares residual formulation by a robust statistics based on [21], [22]. In the literature, the term "residual formulation" is often regarded as a synonym for the term "score function". In [23], [24], robust least-median-squares based approaches were proposed. In [25], a robust bootstrapping M-estimation approach that combines Huber's M-estimation and the bootstrap [26]-[28] was proposed. For further improvement, an adaptive approach that tunes Huber's score function was introduced in [29], [30]. Most of the aforementioned approaches as well as many other robust position estimation approaches can be found in [31]. 
In harsh situations, where NLOS propagation dominates, i.e., a majority of the measurements are outliers, and the probability density function (PDF) of the measurement error is completely unknown, robust position estimation becomes extraordinarily challenging. To the best of our knowledge, those approaches from the first and second categories are not able to work properly under unknown measurement errors. Even if some prior knowledge about the measurement error can be obtained in a training stage, the high computational complexity of these approaches is the main drawback for their practical use. Robust estimation based approaches are favorable in harsh situations, while classical approaches are merely robust for up to $50 \%$ of outliers. In order to achieve more robustness against the NLOS effect, two new fashioned robust estimation approaches have been proposed in [30] and [32] respectively. The gist of the two approaches is to approximate the maximum likelihood estimator (MLE). By following the same design philosophy, we propose in this paper a new robust position estimation approach, which outperforms the existing robust approaches by far.

Our original contributions of this paper are summarized as follows:

- We propose an iterative geolocation algorithm, which alternates between a PDF estimation step and a position estimation step. Starting with a carefully selected initial position estimate, we construct an estimate of the exact measurement error PDF via adaptive kernel density estimation (AKDE) [33], and then formulate an approximate log-likelihood function, from which a refined position estimate is resolved via a quasi-Newton method.

- We present the best achievable geolocation accuracy in terms of Cramér-Rao lower bound (CRLB), which serves as a benchmark for comparison of different robust position estimators.

- We demonstrate the performance of the proposed algorithm in a real-world scenario in Germany. The simulation results show that the proposed algorithm outperforms its competitors by far and behaves nearly optimally for a large sample size.

- We exemplify several practically relevant signal processing applications, to which our algorithm design and CRLB analysis can be directly applied.

The remainder of this paper is organized as follows: In Section II, we introduce the signal model and state the problem at hand. In Section III, we briefly review the robust position estimation approach in [30], followed by our new algorithm. In Section IV, adaptive kernel density estimation is introduced. Theoretical performance analysis is performed in Section V, followed by simulation results which are obtained based on a real-world scenario in Section VI. Finally, Section VII concludes this paper.

\section{Signal Model}

Consider the scenario where $N(N \geq 3)$ base stations (BSs) surround a stationary mobile station (MS) of interest in a wireless cellular network. Let $\left[x_{i}, y_{i}\right]^{T}$ denote the a priori known geographic coordinates of the $i$ th $\mathrm{BS}, i=1,2, \ldots, N$ and let $\boldsymbol{\theta}=[x, y]^{T}$ denote the unknown coordinates of the MS, where the superscript $T$ stands for transpose. For each BS, we obtain $K(K \geq 1)$ range measurement(s) and subsequently relay them to a central site for post-processing [2].

Similar to [17], single path (LOS or NLOS) one-way propagation is considered. Assuming a precise time synchronization between the $\mathrm{BSs}$ and $\mathrm{MS}$, we can express the $k$ th range measurement $r_{i}(k)$ measured at the $i$ th $\mathrm{BS}$ by

$$
r_{i}(k)=\underbrace{\sqrt{\left(x-x_{i}\right)^{2}+\left(y-y_{i}\right)^{2}}}_{h_{i}(\boldsymbol{\theta})}+v_{i}(k),
$$

$k=1,2, \ldots, K$, where $h_{i}(\boldsymbol{\theta})$ represents the Euclidean distance between the MS and the $i$ th BS. ${ }^{1}$ The measurement errors $v_{i}(k)$ are assumed to be independent and identically distributed (i.i.d.) random variables whose PDF is completely unknown to us. In the literature, the measurement error is often reported to have a two-mode mixture PDF which can be written here in a general form as follows:

$$
p_{V}(v)=(1-\varepsilon) \mathcal{N}\left(v ; 0, \sigma_{\mathrm{L}}^{2}\right)+\varepsilon \mathcal{H}(v),
$$

meaning that a random variable $v$ is $\mathcal{N}\left(v ; 0, \sigma_{\mathrm{L}}^{2}\right)$ distributed with probability $(1-\varepsilon)$ in a LOS environment or distributed as $\mathcal{H}(v)$ with probability $\varepsilon$ in an NLOS environment. Here, $\varepsilon$ is referred to as NLOS contamination ratio that quantifies the impact of the NLOS effect, and $\mathcal{N}\left(v ; 0, \sigma_{\mathrm{L}}^{2}\right)$ represents the Gaussian distribution with zero mean and variance $\sigma_{\mathrm{L}}^{2}$. In general, $\mathcal{H}(v)$ has a positive bias and a variance larger than $\sigma_{\mathrm{L}}^{2}$ [6]. It is frequently modeled by a shifted Gaussian distribution $\mathcal{N}\left(v ; \mu_{\mathrm{NL}}, \sigma_{\mathrm{NL}}^{2}\right)$ (e.g., in [6], [30], [32], [34]-[38]), a Rayleigh distribution $\mathcal{R}(v)$ (e.g., in [30], [32], [39], [40]), or an exponential distribution $\mathcal{E}(v)$ (e.g., in [30], [41], [42]), depending on the actual scenario for geolocation. Note that we motivate here the use of the two-mode mixture density model that is often used in a variety of experimentations conducted in mixed LOS/NLOS environments. We stress, however, that the geolocation algorithm we design in the sequel does not assume any model for the measurement errors.

For better readability, we express the signal model in a compact vector form, yielding

$$
\mathbf{r}=\mathbf{h}(\boldsymbol{\theta})+\mathbf{v}
$$

where

$$
\begin{aligned}
\mathbf{r} & =\left[r_{1}(1), \ldots, r_{1}(K), \ldots, r_{N}(1), \ldots, r_{N}(K)\right]^{T}, \\
\mathbf{h}(\boldsymbol{\theta}) & =[\underbrace{h_{1}(\boldsymbol{\theta}), \ldots, h_{1}(\boldsymbol{\theta})}_{K}, \ldots, \underbrace{h_{N}(\boldsymbol{\theta}), \ldots, h_{N}(\boldsymbol{\theta})}_{K}]^{T}, \\
\mathbf{v} & =\left[v_{1}(1), \ldots, v_{1}(K), \ldots, v_{N}(1), \ldots, v_{N}(K)\right]^{T} .
\end{aligned}
$$

Column vectors $\mathbf{r}, \mathbf{h}(\boldsymbol{\theta})$ and $\mathbf{v}$ are all of dimension $N K \times 1$. In the sequel, $r_{m}, h_{m}$ and $v_{m}$ represent the $m$ th element of $\mathbf{r}, \mathbf{h}(\boldsymbol{\theta})$ and $\mathbf{v}$ respectively, with $m=1,2, \ldots, N K$.

Our task is to estimate the MS position $\boldsymbol{\theta}$ based on the available set of range measurements $r$. In contrast to the vast majority of papers available from the literature, we assume that the noise density $p_{V}(v)$ is completely unknown.

\footnotetext{
${ }^{1}$ Range measurement and the corresponding TOA measurement only differ by a constant scaling factor, namely the speed of light in a given medium.
} 


\section{Robust Position Estimation}

For the case that the PDF of the measurement error is completely known, the MLE can be obtained by maximizing the exact log-likelihood function, i.e.,

$$
\begin{aligned}
\hat{\boldsymbol{\theta}}_{\mathrm{MLE}} & =\arg \max _{\boldsymbol{\theta}} \sum_{i=1}^{N} \sum_{k=1}^{K} \log p_{V}\left(r_{i}(k)-h_{i}(\boldsymbol{\theta})\right) \\
& =\arg \max _{\boldsymbol{\theta}} \sum_{m=1}^{N K} \log p_{V}\left(r_{m}-h_{m}\right)
\end{aligned}
$$

where $\log (\cdot)$ denotes the natural logarithm operator for simplicity.

The performance of estimators that rely on maximizing the exact log-likelihood function generally degrades once the true measurement errors deviate from the error model assumption. Since the PDF of the measurement error $p_{V}(v)$ is assumed completely unknown here, finding a robust position estimator whose performance is close to that of the MLE given in (4) is extremely challenging.

The idea we follow in this paper is to jointly combine position estimation with PDF estimation in an iterative process. According to the defintion in [43], the resulting parameter estimation approaches fall in the class of semi-parametric approach when the PDF $p_{V}(v)$ is estimated nonparametrically and the vector parameter $\boldsymbol{\theta}$ to be determined is of finite dimension. The semi-parametric approach, which was initially proposed for robust multiuser detection in impulsive noise channels in [44], has its merits in dealing with the problem where the noise PDF is unknown. In the context of geolocation, however, the design of semi-parametric position estimation approach is more challenging due to the nonlinear signal model in general (e.g., (3) for TOA measurements). Before proceeding with the new approach, however, we first briefly revisit an existing semi-parametric approach for robust position estimation, which was presented in [30].

\section{A. Existing Robust Semi-Parametric Approach}

In [30], the nonlinear signal model is first linearized by squaring both sides of (1) as follows:

$$
\begin{aligned}
r_{i}^{2}(k) & =h_{i}^{2}(\boldsymbol{\theta})+v_{i}^{2}(k)+2 h_{i}(\boldsymbol{\theta}) \cdot v_{i}(k) \\
& =R_{i}+R-2 x_{i} \cdot x-2 y_{i} \cdot y+\tilde{v}_{i}(k),
\end{aligned}
$$

where

$$
\begin{aligned}
R & =x^{2}+y^{2}, \\
R_{i} & =x_{i}^{2}+y_{i}^{2}, \\
\tilde{v}_{i}(k) & =v_{i}^{2}(k)+2 h_{i}(\boldsymbol{\theta}) \cdot v_{i}(k) .
\end{aligned}
$$

Reformulating (5) yields

$$
r_{i}^{2}(k)-R_{i}=-2 x_{i} \cdot x-2 y_{i} \cdot y+R+\tilde{v}_{i}(k) .
$$

Stacking all the terms in a vector, a linear regression model is thus obtained, that is

$$
\tilde{\mathbf{r}}=\mathbf{S} \tilde{\boldsymbol{\theta}}+\tilde{\mathbf{v}}
$$

with the vector notations defined by

$$
\tilde{\mathbf{r}}=\left[\begin{array}{c}
r_{1}^{2}(1)-R_{1} \\
\vdots \\
r_{1}^{2}(K)-R_{1} \\
\vdots \\
r_{N}^{2}(1)-R_{N} \\
\vdots \\
r_{N}^{2}(K)-R_{N}
\end{array}\right], \quad \mathbf{S}=\left[\begin{array}{ccc}
-2 x_{1}, & -2 y_{1}, & 1 \\
& \vdots \\
-2 x_{1}, & -2 y_{1}, & 1 \\
\vdots & \\
-2 x_{N}, & -2 y_{N}, & 1 \\
\vdots & \\
-2 x_{N}, & -2 y_{N}, & 1
\end{array}\right] \text {, }
$$

$\tilde{\mathbf{v}}=\left[\tilde{v}_{1}(1), \ldots, \tilde{v}_{1}(K), \ldots, \tilde{v}_{N}(1), \ldots, \tilde{v}_{N}(K)\right]^{T}$ and $\tilde{\boldsymbol{\theta}}=$ $[x, y, R]^{T}$. Note that $\tilde{\mathbf{r}}$ and $\tilde{\mathbf{v}}$ are both of dimension $N K \times 1$ and $\mathbf{S}$ is of dimension $N K \times 3$. Assuming the elements in $\tilde{\mathbf{v}}$ are i.i.d. random variables with $\operatorname{PDF} p_{\tilde{V}}(\tilde{v})$, although not always true, the log-likelihood function can be expressed by

$$
\sum_{m=1}^{N K} \log p_{\tilde{V}}\left(\tilde{r}_{m}-\mathbf{S}_{m} \tilde{\boldsymbol{\theta}}\right)
$$

where $\mathbf{S}_{m}$ denotes the $m$ th row of matrix $\mathbf{S}$.

Since $p_{\tilde{V}}(\tilde{v})$ is in fact unknown, two conceptually similar iterative algorithms (cf. [30, Table I]) are proposed to resolve an approximate MLE of $\tilde{\boldsymbol{\theta}}$ from

$$
\sum_{m=1}^{N K} \mathbf{S}_{m}^{T} \cdot \varphi\left(\tilde{r}_{m}-\mathbf{S}_{m} \tilde{\boldsymbol{\theta}}\right)=\mathbf{0}
$$

where the score function is defined by

$$
\varphi(\tilde{v})=-\frac{\hat{p}_{\tilde{V}}^{\prime}(\tilde{v})}{\hat{p}_{\tilde{V}}(\tilde{v})}
$$

with $\hat{p}_{\tilde{V}}(\tilde{v})$ denoting the estimated PDF using transformation kernel density estimation (TKDE) and $\hat{p}_{\tilde{V}}^{\prime}(\tilde{v})$ denoting the first order derivative of $\hat{p}_{\tilde{V}}(\tilde{v})$ with respect to $\tilde{v}$. Given an extracted residual vector $\hat{\tilde{\mathbf{v}}}$ on one iteration, TKDE is carried out to give an estimated PDF $\hat{p}_{\tilde{V}}(\tilde{v})$ in the following four steps:

1) Transform the extracted residual vector $\hat{\tilde{\mathbf{v}}}$ via a transformation $\mathbf{z}=t(\hat{\tilde{\mathbf{v}}} ; \zeta)$, where the parameter $\zeta$ steers the shape of this transformation function.

2) Symmetrize the transformed residual vector by $\mathbf{z}_{s}=$ $\left[-\mathbf{z}^{T}, \mathbf{z}^{T}\right]^{T}$.

3) Perform conventional kernel density estimation (KDE) upon $\mathbf{z}_{s}$ and consequently obtain an estimated PDF $\hat{p}_{Z}(z)$.

4) Transform $\hat{p}_{Z}(z)$ back to $\hat{p}_{\tilde{V}}(\tilde{v})$.

Note that the transformation parameter $\zeta$ has to be determined in an optimization procedure prior to the TKDE. Details of the TKDE and the selection of a proper transformation parameter can be found in [30, Appendix A and B], respectively.

Although this semi-parametric approach has shown considerable improvements in the estimation performance as compared to several other salient competitors [30], some issues still remain unsolved. First, an auxiliary parameter $R$ is introduced, but the constraint condition $R=x^{2}+y^{2}$ is not incorporated in the optimization process, which surely leads to a suboptimal solution. The technique proposed in [45] may serve as a powerful tool for remedying this drawback. Secondly, after the linearization, the elements $\tilde{v}_{i}(k)$ for $i=1, \ldots, N$ and 
$k=1, \ldots, K$ in $\tilde{\mathbf{v}}$ are no more identically distributed due to the factor $h_{i}(\boldsymbol{\theta})$ in the expression of $\tilde{v}_{i}(k)$. Thirdly, in [30], a transformation of the original residual vector $\hat{\tilde{\mathbf{v}}}$ has to be conducted but there is no well established rule underpinning the selection of a parametric transformation function $t(\hat{\tilde{\mathbf{v}}} ; \zeta)$ as well as an appropriate interval $\left[\zeta_{L}, \zeta_{U}\right]$ for optimizing $\zeta$, which are crucial to the PDF estimation. As illustrated in [30, Fig. 7], a wrongly selected transformation parameter $\zeta$ may severely degrade the geolocation accuracy. For sake of avoiding these drawbacks, we propose a new approach below.

\section{B. Proposed Approach}

The newly proposed approach is also a semi-parametric approach according to the definition in [43]. In order to distinguish between the two semi-parametric approaches, we refer to the new approach as "robust iterative nonparametric (RIN) approach" with the term "nonparametric" indicating the fact that the measurement error $\operatorname{PDF} p_{V}(v)$ is estimated nonparametrically, and follow the name "semi-parametric approach" for the existing approach in the remaining parts of this paper.

The main features of the new approach as compared to the existing semi-parametric approach in [30] are highlighted as follows. Firstly, the proposed algorithm directly uses the nonlinear signal model. As a consequence, evaluation of the constraint condition $R=x^{2}+y^{2}$ is avoided and the elements in $\mathbf{v}$ are i.i.d.. ${ }^{2}$ Secondly, TKDE is replaced by nonparametric adaptive kernel density estimation (AKDE) to obtain an estimate of the measurement error PDF. This has the advantage that the parameters required for constructing a density estimator are now set adaptively and fully automatically as compared to the TKDE. Thirdly, a quasi-Newton method is employed to resolve a position estimate from the approximate log-likelihood function, which is derived based on the a priori calculated PDF estimate. The key steps of the RIN position estimation approach are summarized in Algorithm 1.

It is noteworthy that,

- the initial estimate $\hat{\boldsymbol{\theta}}^{(0)}$ is set by the first two entries of the least-squares solution of (10), i.e., by $\left[\tilde{x}_{\mathrm{LS}}, \tilde{y}_{\mathrm{LS}}\right]^{T}$ of

$$
\tilde{\boldsymbol{\theta}}_{\mathrm{LS}}=\left[\tilde{x}_{\mathrm{LS}}, \tilde{y}_{\mathrm{LS}}, \tilde{R}_{\mathrm{LS}}\right]^{T}=\left(\mathbf{S}^{T} \mathbf{S}\right)^{-1} \mathbf{S}^{T} \tilde{\mathbf{r}}
$$

- the approximated noise density $\hat{p}_{V}^{(j)}(v)$ is composed of a sum of Gaussian kernels as follows

$\hat{p}_{V}^{(j)}(v)=\frac{1}{N K} \sum_{m=1}^{N K} \frac{1}{\sqrt{2 \pi} w^{(j)} \lambda_{m}^{(j)}} \exp \left[-\frac{\left(v-\hat{v}_{m}^{(j)}\right)^{2}}{2\left(w^{(j)} \lambda_{m}^{(j)}\right)^{2}}\right]$

where $\hat{v}_{m}^{(j)}$ denotes the $m$ th element of the residual vector $\hat{\mathbf{v}}^{(j)} ; w^{(j)}$ denotes the window width; and $\lambda_{m}^{(j)}$, $m=1,2, \ldots, N K$ denote the local bandwidth factors calculated in the $j$ th iteration;

\footnotetext{
${ }^{2}$ It is necessary to stress here again that we work with the nonlinear signal model in (3), where the entries in $\mathbf{v}$ are i.i.d., for the new algorithm design and CRLB analysis, while the existing semi-parametric approach [30] works with the linearized signal model in (10), where the entries in $\tilde{\mathbf{v}}$ are not i.i.d in general from our analysis.
}

$\overline{\text { Algorithm } 1 \text { Robust Iterative Nonparametric (RIN) Position }}$ Estimation Approach

Step 1-Initialization: Define the convergence tolerance $\Delta$ and the maximum number of iterations $N_{\mathrm{Itr}}^{\max }$; Set the iteration index $j=0$; Choose an initial guess $\hat{\boldsymbol{\theta}}^{(0)}$ of the position parameter $\boldsymbol{\theta}$ to be determined.

Step 2-Perform Joint Estimation: In the $j$ th $(j \geq 1)$ iteration, sequentially do:

1) Determine the residual vector $\hat{\mathbf{v}}^{(j)}=\mathbf{r}-\mathbf{h}\left(\hat{\boldsymbol{\theta}}^{(j-1)}\right)$.

2) Construct an estimate of the true probability density function $p_{V}(v), \hat{p}_{V}^{(j)}(v)$, from $\hat{\mathbf{v}}^{(j)}$ via the nonparametric AKDE described in Algorithm 3.

3) Approximate the exact log-likelihood function by

$$
l l^{(j)}(\boldsymbol{\theta})=\sum_{i=1}^{N} \sum_{k=1}^{K} \log \hat{p}_{V}^{(j)}\left(r_{i}(k)-h_{i}(\boldsymbol{\theta})\right) .
$$

4) Define the negative of the approximate log-likelihood function by $g^{(j)}(\boldsymbol{\theta})=-l l^{(j)}(\boldsymbol{\theta})$ and update the position estimate by minimizing $g^{(j)}(\boldsymbol{\theta})$, more specifically,

$$
\hat{\boldsymbol{\theta}}^{(j)}=\arg \min _{\boldsymbol{\theta}} g^{(j)}(\boldsymbol{\theta}),
$$

using (for instance) Algorithm 2.

Step 3-Convergence Check: If $\left\|\hat{\boldsymbol{\theta}}^{(j)}-\hat{\boldsymbol{\theta}}^{(j-1)}\right\|<\Delta$ or the maximum number of iterations $N_{\mathrm{Itr}}^{\max }$ is reached, then we terminate the algorithm; otherwise we update the iteration index $j \leftarrow j+1$ and return to Step 2 .

- the cost function $g^{(j)}(\boldsymbol{\theta})$ is given by

$$
\begin{aligned}
g^{(j)}(\boldsymbol{\theta})= & -\sum_{i=1}^{N} \sum_{k=1}^{K} \log \frac{1}{N K} \sum_{m=1}^{N K} \frac{1}{\sqrt{2 \pi} w^{(j)} \lambda_{m}^{(j)}} \\
& \times \exp \left[-\frac{\left(r_{i}(k)-h_{i}(\boldsymbol{\theta})-\hat{v}_{m}^{(j)}\right)^{2}}{2\left(w^{(j)} \lambda_{m}^{(j)}\right)^{2}}\right] ;
\end{aligned}
$$

- the operator $\|\cdot\|$ denotes the $\mathcal{L}_{2}$ norm.

Many numerical methods can be utilized to solve the minimization problem formulated in (15), e.g., the NewtonRaphson method [46], quasi-Newton method [47], and the expectation maximization method [48]. Alternatively, it is safest to perform a two-dimensional (2-D) grid search in the vicinity of a good initial guess [49]. But the drawback lies in the higher computational complexity. In this paper, we use the Broyden-Fletcher-Goldfarb-Shanno (BFGS) quasi-Newton method to minimize the nonlinear cost function $g(\boldsymbol{\theta})$, since it guarantees downhill progress towards the local minimum in each Newton step [50]. The key steps of the BFGS quasiNewton method are listed in Algorithm 2. It is noteworthy to mention that the iteration index $j$ of $g^{(j)}(\boldsymbol{\theta})$ is discarded for brevity in Algorithm 2.

The reasons that we choose the least-squares solution as the initial value in Algorithm 1 are due to its simplicity and rather low computational complexity [6]. However, in the case where the least-squares solution may largely lose its accuracy, we have to resort to a more sophisticated strategy in which we use several candidate initial values and finally choose the one 
leading to the maximal value of the approximate log-likelihood $l l^{(1)}(\boldsymbol{\theta})$ (cf.(14)) after the first iteration of Algorithm 1. ${ }^{3}$ The disadvantage of such an approach is the increase in computational complexity. In the sequel, we merely test the leastsquares solution as the initial guess. In Section VI, simulations confirm the performance of this simpler initialization strategy.

It is conspicuous from Algorithm 1 that the parametric position estimation and the nonparametric PDF estimation are tightly combined in an iteration process. Intuitively, the improved position estimate will lead to a refined PDF estimate and vice versa so that at the convergence of this iterative algorithm a good estimation performance can be achieved. However, it remains difficult, even asymptotically, to theoretically analyze the performance difference between the new position estimator and the corresponding MLE (cf. (4)) in terms of bias and root mean squared error (RMSE). While the MLE is known to be asymptotically efficient under some regularity conditions, see [49, Theorem 7.3], this is not easy to show for the new position estimator, due to the difficulties in quantifying how well the true $\operatorname{PDF} p_{V}(v)$ can be approximated by its estimate $\hat{p}_{V}(v)$ for a given number of observations. But we still believe that the performance of the new position estimator can be very close to that of the MLE when the number of range measurements is sufficiently large. In this paper, we evaluate the actual performance of the new position estimator in a large-scale Monte Carlo simulation.

Algorithm 2 BFGS quasi-Newton method with a cubic line search

1) Set $l=0$ and obtain a search direction $\mathbf{s}_{l}=-\mathbf{H}_{l}$. $\nabla_{\boldsymbol{\theta}} g\left(\hat{\boldsymbol{\theta}}_{l}\right)$, where $\nabla_{\boldsymbol{\theta}} g\left(\hat{\boldsymbol{\theta}}_{l}\right)$ is the gradient of the cost function $g(\boldsymbol{\theta})$ evaluated at $\hat{\boldsymbol{\theta}}_{l}$. The initial value $\hat{\boldsymbol{\theta}}_{0}$ is set to $\hat{\boldsymbol{\theta}}^{(j-1)}$ on the $j$ th $(j \geq 1)$ iteration of Algorithm 1 .

2) Find the step size $\alpha_{l}$ along the direction $\mathbf{s}_{l}$ via cubic line search introduced in [51, Algorithm 3.5 and 3.6].

3) Update the estimate by $\hat{\boldsymbol{\theta}}_{l+1}=\hat{\boldsymbol{\theta}}_{l}+\alpha_{l} \mathbf{s}_{l}$.

4) Set $\boldsymbol{\delta}_{l}=\alpha_{l} \mathbf{s}_{l}$ and $\boldsymbol{\gamma}_{l}=\nabla_{\boldsymbol{\theta}} g\left(\hat{\boldsymbol{\theta}}_{l+1}\right)-\nabla_{\boldsymbol{\theta}} g\left(\hat{\boldsymbol{\theta}}_{l}\right)$.

5) Update the approximate Hessian matrix by

$\mathbf{H}_{l+1}=\mathbf{H}_{l}+\left(1+\frac{\boldsymbol{\gamma}_{l}^{T} \mathbf{H}_{l} \boldsymbol{\gamma}_{l}}{\boldsymbol{\delta}_{l}^{T} \boldsymbol{\gamma}_{l}}\right) \frac{\boldsymbol{\delta}_{l} \boldsymbol{\delta}_{l}^{T}}{\boldsymbol{\delta}_{l}^{T} \gamma_{l}}-\left(\frac{\boldsymbol{\delta}_{l} \boldsymbol{\gamma}_{l}^{T} \mathbf{H}_{l}+\mathbf{H}_{l} \boldsymbol{\gamma}_{l} \boldsymbol{\delta}_{l}^{T}}{\boldsymbol{\delta}_{l}^{T} \gamma_{l}}\right)$

Note that, the initial approximate Hessian matrix $\mathbf{H}_{0}$ is set to an identity matrix $\mathbf{I}_{2}$.

6) If $\left\|\hat{\boldsymbol{\theta}}_{l+1}-\hat{\boldsymbol{\theta}}_{l}\right\|<\kappa$, then stop; otherwise update the iteration index $l \leftarrow l+1$ and return to step 2 .

\section{AdAPtiVE KeRnel DEnsity Estimation}

Kernel density estimation (KDE) is a nonparametric approach for estimating the PDF based on a given set of observations [33]. The key idea of KDE is to approximate the desired PDF by a linear combination of kernel functions with carefully

\footnotetext{
${ }^{3}$ We can employ, for instance, the position estimates obtained from the robust M-estimation approach [29], [30] and the robust semi-parametric estimation approach [30] as well as the grid points in the vicinity of them. However, the computational cost highly depends on the total number of the grid points.
}

chosen bandwidths. Amongst a large number of variations of this kind, we employ here an adaptive kernel density estimation approach, which gives overall good performance in estimating a long-tailed and/or multi-modal PDF [33].

Assuming that we have a set of $N K$ i.i.d. observations $\mathbf{v}=\left\{v_{1}, v_{2}, \ldots, v_{N K}\right\}$ generated from a continuous univariate distribution with probability density function $p_{V}(v)$, the steps required for constructing an adaptive kernel density estimator $\hat{p}_{V}(v)$ are demonstrated in Algorithm 3.

$$
\begin{aligned}
& \text { Algorithm } 3 \text { Adaptive Kernel Density Estimator } \\
& \hline \text { 1) Find a pilot density estimator } \\
& \qquad \hat{p}_{0}(v)=\frac{1}{N K} \sum_{m=1}^{N K} \frac{1}{w_{0}} G\left(\frac{v-v_{m}}{w_{0}}\right)
\end{aligned}
$$

where $G(\cdot)$ denotes a standard Gaussian kernel, $w_{0}=$ $0.79 \cdot \operatorname{Iqr}\{\mathbf{v}\} \cdot(N K)^{-1 / 5}$ denotes an initial global bandwidth and $\operatorname{Iqr}\{\mathbf{v}\}$ denotes the interquartile range of $\mathbf{v}=\left\{v_{1}, v_{2}, \ldots, v_{N K}\right\}$.

2) Define local bandwidths $\lambda_{m}, m=1,2, \ldots, N K$ by

$$
\lambda_{m}=\left(\hat{p}_{0}\left(v_{m}\right) /\left[\prod_{m=1}^{N K} \hat{p}_{0}\left(v_{m}\right)\right]^{\frac{1}{N K}}\right)^{-\beta}
$$

where the sensitivity parameter $\beta$ is set to 0.5 as suggested in [52].

3) An adaptive kernel density estimator $\hat{p}_{V}(v)$ is finally constructed by

$$
\hat{p}_{V}(v)=\frac{1}{N K} \sum_{m=1}^{N K} \frac{1}{w \lambda_{m}} G\left(\frac{v-v_{m}}{w \lambda_{m}}\right)
$$

where the global bandwidth $w$ is determined using the least-squares cross-validation technique [33].

\section{Theoretical Performance Metrics For GEOLOCATION ACCURACY}

In this section, different performance metrics are presented that can be used to assess the best achievable performance of position estimators in harsh LOS/NLOS environments. Here, it is worth noting that for the theoretical performance analysis, $p_{V}(v)$ is assumed to be known.

\section{A. Cramér-Rao Lower Bound}

It is well known that the covariance matrix of any unbiased estimator of an unknown vector parameter is lower bounded by the Cramér-Rao lower bound [49], [17]. In the following, CRLB analysis will be carried out based on our nonlinear signal model in (3). Let $\hat{\boldsymbol{\theta}}=[\hat{x}, \hat{y}]^{T}$ denote an unbiased position estimator of a deterministic vector position parameter $\boldsymbol{\theta}=[x, y]^{T}$ and let $\operatorname{Cov}(\hat{\boldsymbol{\theta}})$ denote the covariance matrix of $\hat{\boldsymbol{\theta}}$. Assuming that certain regularity conditions are fulfilled [53], we have

$$
\begin{aligned}
\operatorname{Cov}(\hat{\boldsymbol{\theta}}) & =\mathbb{E}_{p(\mathbf{r} \mid \boldsymbol{\theta})}\left\{(\hat{\boldsymbol{\theta}}-\boldsymbol{\theta})(\hat{\boldsymbol{\theta}}-\boldsymbol{\theta})^{T}\right\} \\
& \succeq \mathcal{F}^{-1}(\boldsymbol{\theta}) \equiv \mathrm{CRLB}
\end{aligned}
$$


where $\mathcal{F}(\boldsymbol{\theta})$ denotes Fisher information matrix (FIM), and $\mathbf{A} \succeq \mathbf{B}$ means that the matrix difference $\mathbf{A}-\mathbf{B}$ is positive semidefinite. Let $\nabla_{\boldsymbol{\theta}}=[\partial / \partial x, \partial / \partial y]^{T}$ denote the gradient operator, and let $\Delta_{\boldsymbol{\theta}}^{\boldsymbol{\theta}}=\nabla_{\boldsymbol{\theta}} \nabla_{\boldsymbol{\theta}}^{T}$ denote the Laplace operator. Then, FIM can be expressed as

$$
\mathcal{F}(\boldsymbol{\theta})=\mathbb{E}_{p(\mathbf{r} \mid \boldsymbol{\theta})}\left\{-\Delta_{\boldsymbol{\theta}}^{\boldsymbol{\theta}} \log p(\mathbf{r} \mid \boldsymbol{\theta})\right\}
$$

where the expectation is taken with respect to $p(\mathbf{r} \mid \boldsymbol{\theta})$, namely the PDF of $\mathbf{r}$ parameterized in $\boldsymbol{\theta}$. For nonlinear models with non-Gaussian error $\mathbf{v}$ in (3), closed-form expressions for FIM are generally not available. Here, Monte Carlo integration techniques [54] can be used to numerically approximate the expectation given in (24). Instead of performing Monte Carlo integration directly on (24), we suggest the following procedure. Often, it is more convenient to express FIM as follows

$$
\mathcal{F}(\boldsymbol{\theta})=\mathbb{E}_{p(\mathbf{r} \mid \boldsymbol{\theta})}\left\{\frac{\nabla_{\boldsymbol{\theta}} p(\mathbf{r} \mid \boldsymbol{\theta}) \nabla_{\boldsymbol{\theta}}^{T} p(\mathbf{r} \mid \boldsymbol{\theta})}{p(\mathbf{r} \mid \boldsymbol{\theta})^{2}}\right\} .
$$

It was shown in [55], [56] that for nonlinear models given by (3), FIM in (25) can be reformulated as

$$
\mathcal{F}(\boldsymbol{\theta})=\mathcal{H}(\boldsymbol{\theta}) \cdot \mathcal{I}_{\mathbf{v}} \cdot \mathcal{H}^{T}(\boldsymbol{\theta})
$$

where

$$
\begin{aligned}
& \mathcal{H}(\boldsymbol{\theta})=\nabla_{\boldsymbol{\theta}} \mathbf{h}(\boldsymbol{\theta}) \\
& =\left[\begin{array}{l}
\underbrace{\frac{x-x_{1}}{h_{1}(\boldsymbol{\theta})}, \cdots, \frac{x-x_{1}}{h_{1}(\boldsymbol{\theta})}}_{\text {Krepetitions }}, \cdots, \underbrace{\frac{x-x_{N}}{h_{N}(\boldsymbol{\theta})}, \cdots, \frac{x-x_{N}}{h_{N}(\boldsymbol{\theta})}}_{K \text { repetitions }} \\
\underbrace{\frac{y-y_{1}}{h_{1}(\boldsymbol{\theta})}, \cdots, \frac{y-y_{1}}{h_{1}(\boldsymbol{\theta})}}_{K \text { repetitions }}, \cdots, \underbrace{\frac{y-y_{N}}{h_{N}(\boldsymbol{\theta})}, \cdots, \frac{y-y_{N}}{h_{N}(\boldsymbol{\theta})}}_{K \text { repetitions }}
\end{array}\right]
\end{aligned}
$$

and

$$
\mathcal{I}_{\mathbf{v}}=\mathbb{E}_{p_{\mathbf{v}}(\mathbf{v})}\left\{\frac{\left[\nabla_{\mathbf{v}} p_{\mathbf{v}}(\mathbf{v})\right] \cdot\left[\nabla_{\mathbf{v}} p_{\mathbf{v}}(\mathbf{v})\right]^{T}}{\left[p_{\mathbf{v}}(\mathbf{v})\right]^{2}}\right\} .
$$

In Appendix A, it is proven that as long as the elements in $\mathbf{v}$ are i.i.d., the matrix $\mathcal{I}_{\mathbf{v}}$ is a diagonal matrix of the form

$$
\mathcal{I}_{\mathbf{v}}=\mathcal{I}_{v} \cdot \mathbf{I}_{N K}
$$

where $\mathbf{I}_{N K}$ is an identity matrix of dimension $N K \times N K$, and $\mathcal{I}_{v}$ is calculated by

$$
\begin{aligned}
\mathcal{I}_{v} & =\mathbb{E}_{p_{V}(v)}\left\{\frac{\left[\nabla_{v} p_{V}(v)\right]^{2}}{p_{V}^{2}(v)}\right\} \\
& =\int \frac{\left[\nabla_{v} p_{V}(v)\right]^{2}}{p_{V}^{2}(v)} p_{V}(v) \mathrm{d} v
\end{aligned}
$$

and referred to as intrinsic accuracy [57]. For most of the measurement error distributions, it is difficult to derive $\mathcal{I}_{v}$ in closed form. A notable exception, however, is the Gaussian distribution $\mathcal{N}\left(v ; \mu_{v}, \sigma_{v}^{2}\right)$ and in this case $\mathcal{I}_{v}=\sigma_{v}^{-2}$. In the other cases, the integral in (30) can be approximated using Monte Carlo integration [54], yielding

$$
\mathcal{I}_{v} \approx \frac{1}{N_{C}} \sum_{n=1}^{N_{C}} \frac{\left[\nabla_{v} p_{V}\left(v^{(n)}\right)\right]^{2}}{p_{V}^{2}\left(v^{(n)}\right)}
$$

where $v^{(n)}, n=1,2, \ldots, N_{C}$ are $N_{C}$ i.i.d. samples generated from $p_{V}(v)$. It is obvious from (29) that for a specified $p_{V}(v)$, only one Monte Carlo integration has to be performed to compute $\mathcal{I}_{\mathbf{v}}$ if the elements in the vector $\mathbf{v}$ are i.i.d., and this $\mathcal{I}_{\mathrm{v}}$ can be used to compute the CRLB for several different MS positions. This comes with a considerable reduction in computational complexity as compared to performing Monte Carlo integration directly on (25).

\section{B. Bias and Root Mean Squared Error}

In geolocation applications, it is desirable to design an unbiased estimator with RMSE as small as possible [58]. The bias of a position estimator is defined here by

$$
\operatorname{Bias}(\boldsymbol{\theta})=\mathbb{E}_{p(\mathbf{r} \mid \boldsymbol{\theta})}\{\hat{\boldsymbol{\theta}}\}-\boldsymbol{\theta}
$$

and the RMSE, frequently interpreted as the geolocation accuracy of a position estimator, is related to the obtained CRLB through

$$
\begin{aligned}
\mathrm{RMSE} & \equiv \sqrt{\mathbb{E}_{p(\mathbf{r} \mid \boldsymbol{\theta})}\left\{(\hat{x}-x)^{2}+(\hat{y}-y)^{2}\right\}} \\
& =\sqrt{\operatorname{tr}(\operatorname{Cov}(\hat{\boldsymbol{\theta}}))} \geq \sqrt{\operatorname{tr}\left(\mathcal{F}^{-1}(\boldsymbol{\theta})\right)} \equiv \mathrm{CRLB}_{\mathrm{pos}}
\end{aligned}
$$

where $\operatorname{tr}(\cdot)$ denotes the trace of a matrix, and $\mathrm{CRLB}_{\text {pos }}$ can be interpreted as the best achievable RMSE of an unbiased position estimator.

\section{Estimation Efficiency}

Here, it is more convenient to re-define the efficiency of an unbiased position estimator by

$$
\eta=\frac{\mathrm{CRLB}_{\mathrm{pos}}}{\mathrm{RMSE}}=\sqrt{\frac{\operatorname{tr}\left(\mathcal{F}^{-1}(\boldsymbol{\theta})\right)}{\operatorname{tr}(\operatorname{Cov}(\hat{\boldsymbol{\theta}}))} .}
$$

It follows from (33) that $0 \leq \eta \leq 1$. If a position estimator is unbiased and simultaneously attains $\eta=1$, then it is referred to as an efficient position estimator. As it is well known, the MLE obtained from (4) is asymptotically efficient—unbiased and $\eta=1-$ as the number of measurements goes to infinity.

\section{Geometric Dilution of Precision}

Another important metric is called geometric dilution of precision (GDOP), which is used to describe the influence of BSsMS geometry on the relationship between the measurement error and geolocation accuracy [59]. Since we have assumed identical variance of the measurement errors at different BSs, GDOP is easily calculated by

$$
\mathrm{GDOP}=\sqrt{\frac{\operatorname{tr}(\operatorname{Cov}(\hat{\boldsymbol{\theta}}))}{\sigma_{v}^{2}}}
$$

where $\sigma_{v}^{2}$ denotes the variance of the measurement error PDF, $p_{V}(v)$. For an efficient position estimator, (35) becomes

$$
\mathrm{GDOP}=\frac{\mathrm{CRLB}_{\mathrm{pos}}}{\sigma_{v}} .
$$

It was reported in [31] that GDOP values smaller than three imply well-suited geometry, whereas those larger than six imply a deficient geometry. 
TABLE I

Simulation PARAMETERS

\begin{tabular}{|l|l|l|}
\hline Parameter & Section & Value \\
\hline$N$ & VI & 8 \\
\hline \multirow{3}{*}{$K$} & VI-A & 1 sample \\
& VI-B, C, E & 20 samples \\
& VI-D & $5: 5: 75$ samples \\
\hline \multirow{2}{*}{} & VI-A, C, D & 0.5 \\
& VI-B, E & $0: 0.1: 1$ \\
\hline$\sigma_{\mathrm{L}}$ & VI & 55 meter $(m)$ \\
\hline$\mu_{\mathrm{NL}}$ & VI & $380 m$ \\
\hline$\sigma_{\mathrm{NL}}$ & VI & $120 m$ \\
\hline$\Delta$ & VI-B, C, D, E & $0.1 m$ \\
\hline$\kappa$ & VI-B, C, D, E & $10^{-6} m$ \\
\hline$N_{\text {Itr }}^{\max }$ & VI-B, C, D, E & 20 iterations \\
\hline$N_{C}$ in (31) & VI-A, B, D & 50,000 samples \\
\hline \multirow{2}{*}{$M C$} & VI-B, E & 2500 trials \\
& VI-C & 1 trial \\
& VI-D & 1000 trials \\
\hline
\end{tabular}

\section{Simulations}

We consider geolocation of a stationary MS in a cellular radio network which comprises $N$ BSs with fixed and known geographic coordinates. In order to be as realistic as possible, the BS locations are taken from an operating cellular radio network in a German city center [36], [60]. The geometry of the BSs as well as the approximate location of the city center is shown in Fig. 1. The BS antennae are generally deployed on rooftops, and the city center can be characterized as urban with multi-storey buildings and narrow streets. Field trials conducted in this city have revealed that the TOA measurement error can be well approximated by a Gaussian mixture distribution [36]. In the simulations, we generate range measurement errors from the following two-mode Gaussian mixture distribution

$$
p_{V}(v)=(1-\varepsilon) \mathcal{N}\left(v ; 0, \sigma_{\mathrm{L}}^{2}\right)+\varepsilon \mathcal{N}\left(v ; \mu_{\mathrm{NL}}, \sigma_{\mathrm{NL}}^{2}\right)
$$

as mentioned in Section II. The parameters of the Gaussian mixture as well as all other simulation parameters are summarized in Table I.

The newly proposed robust iterative nonparametric estimator is compared to the following position estimators:

- Least-squares estimator, cf. (16)

- Robust M-estimator [29], [30]

- Robust semi-parametric estimator [30]

- Robust nonparametric estimator [32]

- MLE with known $p_{V}(v)$

The first three approaches are all developed under the linear regression model in (10). In the robust M-estimation approach, the clipping point $c$ of Huber's score function $\psi(\tilde{v} ; c)$ (cf. [30, (7)]) is adaptively calculated by $c=0.6 /(1.483 \cdot \operatorname{mad}(\hat{\tilde{\mathbf{v}}}))$, where $\hat{\tilde{\mathbf{v}}}$ denotes the a priori extracted residual vector in each iteration and $\operatorname{mad}(\cdot)$ denotes the median absolute deviation. In the semi-parametric approach [30], we choose the same transformation function $t(\hat{\tilde{\mathbf{v}}} ; \zeta)$ and search interval $\left[\zeta_{L}=0.9, \zeta_{U}=\right.$ $1]$ as suggested therein. The robust nonparametric approach follows Algorithm 1, except that the number of iterations is constrained to one. By assuming $p_{V}(v)$ to be known, we resolve the MLE, serving as a benchmark for comparison, from (4) via the quasi-Newton method (cf. Algorithm 2) with

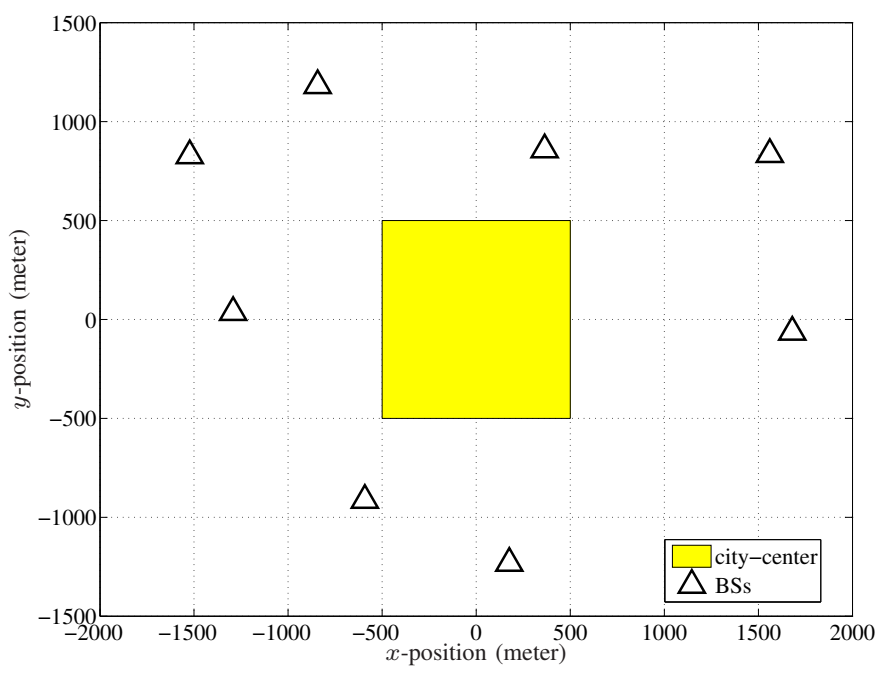

Fig. 1. 2-D illustration of the geometry of BSs and the city center area in a real-world scenario in Germany.

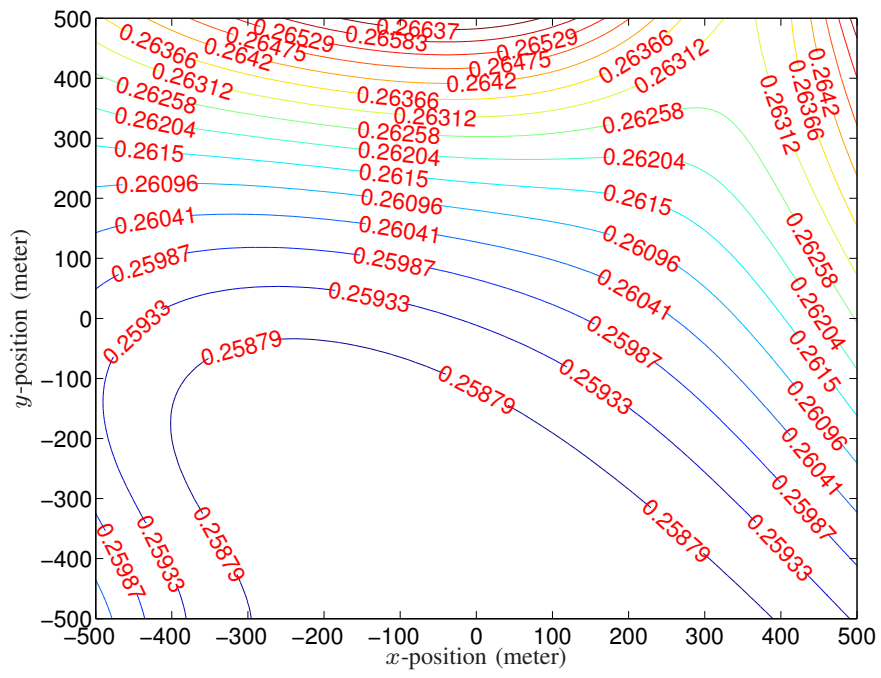

Fig. 2. GDOP values measured for an efficient position estimator in the city center area with $K=1$ and $\varepsilon=0.5$.

the initial guess set by the true MS position. Apart from the MLE, the rest of estimators do not assume $p_{V}(v)$ to be known a priori.

\section{A. Geometric Dilution of Precision}

In the first simulation, GDOP values are computed according to (36) (i.e., assuming an efficient position estimator) for various different positions in the city center area as illustrated in Fig. 1. In this simulation, the number of range measurements $K$ obtained at each BS is set to one and the NLOS contamination ratio $\varepsilon$ is set to 0.5 . By following the results derived in [50, Section 1.4.16], $\sigma_{v}$ in (36) is calculated by

$$
\sigma_{v}=\sqrt{(1-\varepsilon) \cdot \sigma_{\mathrm{L}}^{2}+\varepsilon \cdot \sigma_{\mathrm{NL}}^{2}+\varepsilon \cdot(1-\varepsilon) \cdot \mu_{\mathrm{NL}}^{2}} .
$$

The resulting GDOP values are shown in Fig. 2, and they indicate a well-suited geometry of the BSs for locating a MS in 


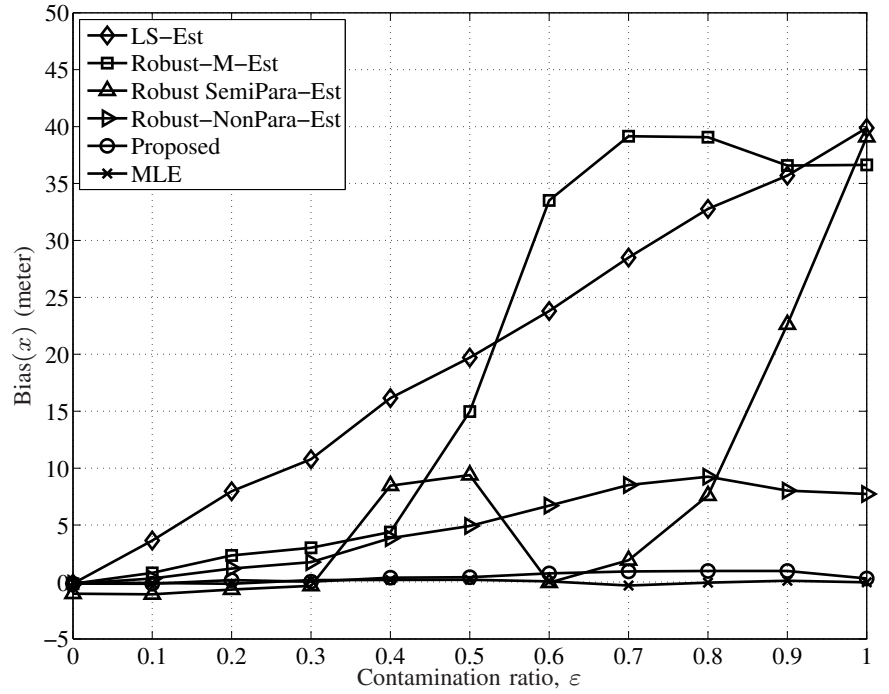

(a)

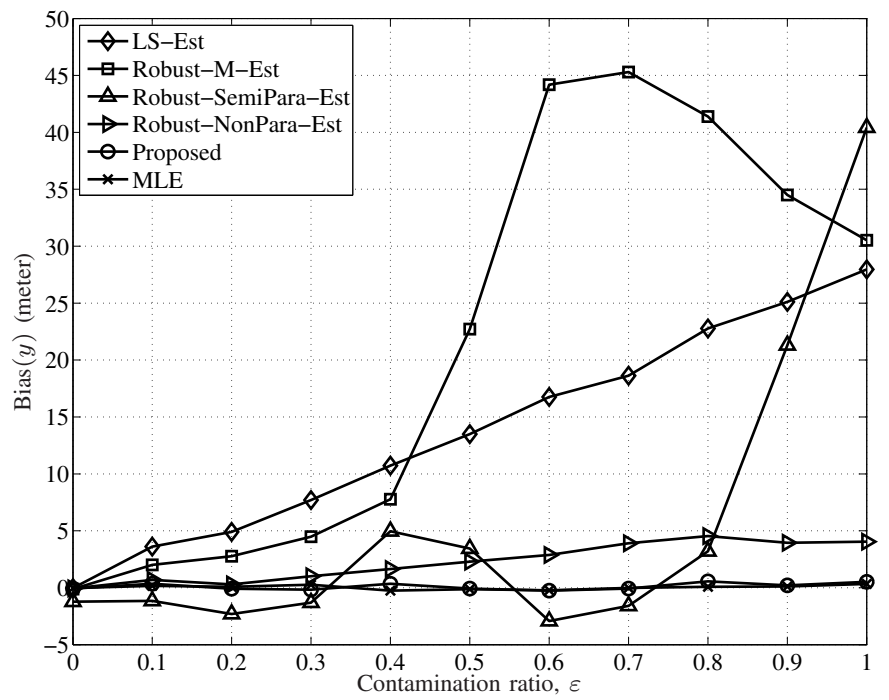

(b)

Fig. 3. Bias (assuming a fixed set of geographic coordinates $[x=$ $0.25 \mathrm{~km}, y=0.25 \mathrm{~km}]^{T}$ ) of the different position estimators versus NLOS contamination ratio $\varepsilon$ with $K=20$. (a) describes the bias in $x$-position; and (b) describes the bias in $y$-position.

the city center area. When we further increase $K$ or decrease $\varepsilon$, better GDOP values are achievable. A proper discussion on the impact of BSs-MS geometry on the geolocation accuracy is out of the scope of our paper. Interested readers are referred to $[8$, Chapter 13] and references therein.

\section{B. Bias, RMSE and CRLB}

In this simulation, the different position estimators are evaluated in terms of bias and RMSE. To this end, the bias and the RMSE of the different position estimators are evaluated in a large-scale Monte Carlo simulation with $M C=2500$ trials. Two examples are investigated in the sequel.

The first example assumes that the MS is located at $[x=$ $250 \mathrm{~m}, y=250 \mathrm{~m}]^{T}$ and the number of range measurements at each BS is $K=20$ samples. The bias and RMSE of the

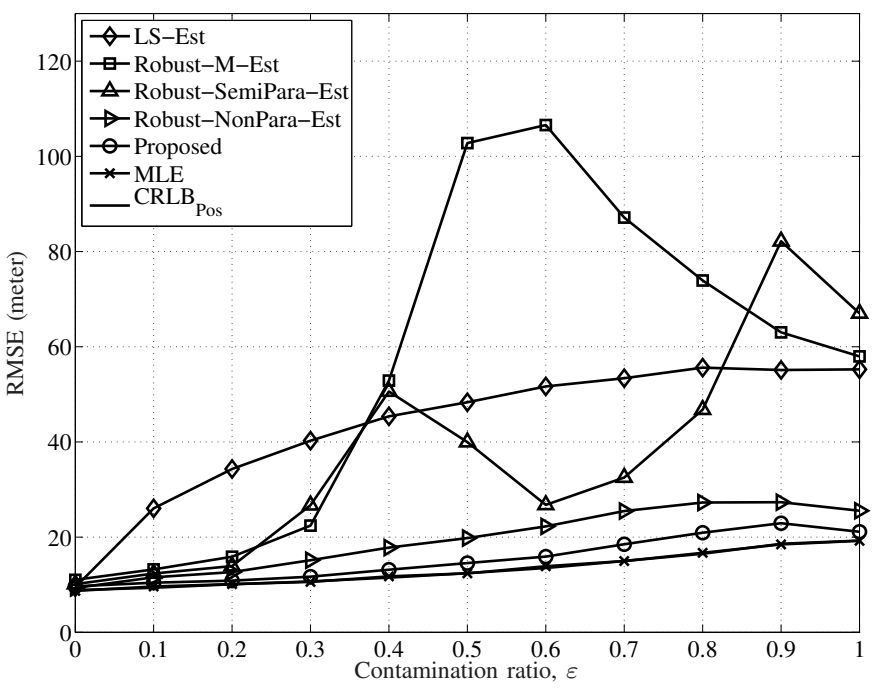

Fig. 4. RMSE (assuming a fixed set of geographic coordinates $[x=$ $0.25 \mathrm{~km}, y=0.25 \mathrm{~km}]^{T}$ ) of the different position estimators versus NLOS contamination ratio $\varepsilon$ with $K=20$.

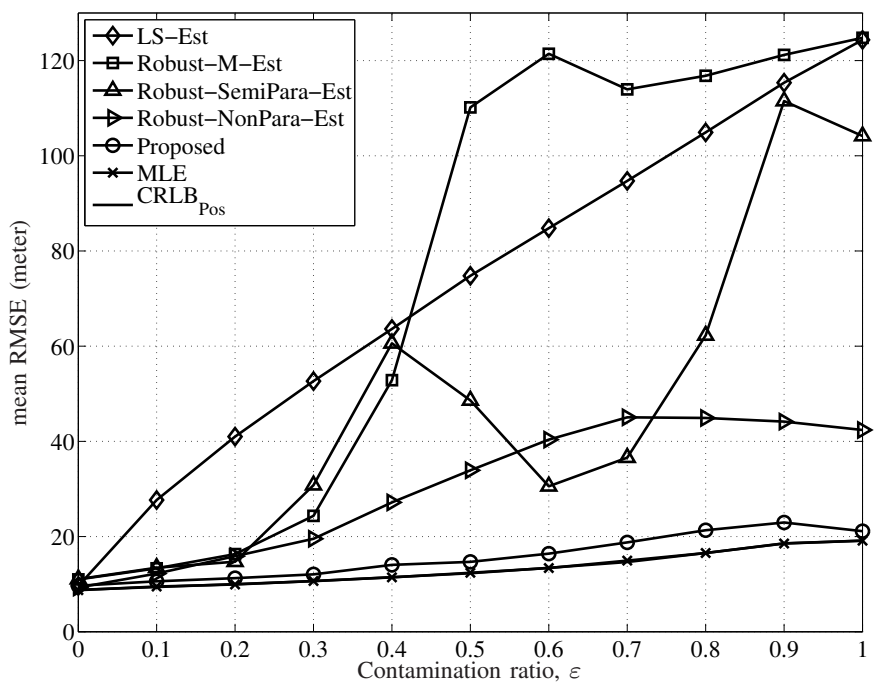

Fig. 5. Mean RMSE (over 2000 sets of geographic coordinates uniformly generated from the city center area) of the different position estimators versus NLOS contamination ratio $\varepsilon$ with $K=20$.

aforementioned position estimators are evaluated as a function of the NLOS contamination ratio $0 \leq \varepsilon \leq 1$. In Fig. 3 and Fig. 4 the results for the bias and RMSE are shown. The performance lower bound $\mathrm{CRLB}_{\text {pos }}$ is calculated according to (33) and shown here to serve as a benchmark for comparison of different RMSE curves. ${ }^{4}$ The results mainly reveal the following two aspects. Firstly, the different robust position estimators perform similarly (close to the MLE) when the NLOS contamination ratio $\varepsilon$ is close to zero. The reasons are

${ }^{4}$ When $\varepsilon=0$ or 1, FIM has the following analytical form:

$$
\begin{array}{cl}
\mathcal{F}(\boldsymbol{\theta})=\sigma_{\mathrm{L}}^{-2} \cdot \mathcal{H}(\boldsymbol{\theta}) \mathcal{H}^{T}(\boldsymbol{\theta}), & \text { for } \varepsilon=0, \\
\mathcal{F}(\boldsymbol{\theta})=\sigma_{\mathrm{NL}}^{-2} \cdot \mathcal{H}(\boldsymbol{\theta}) \mathcal{H}^{T}(\boldsymbol{\theta}), & \text { for } \varepsilon=1 .
\end{array}
$$

Therefore, there is no need to perform a Monte Carlo integration for approximating FIM for these two special cases. 


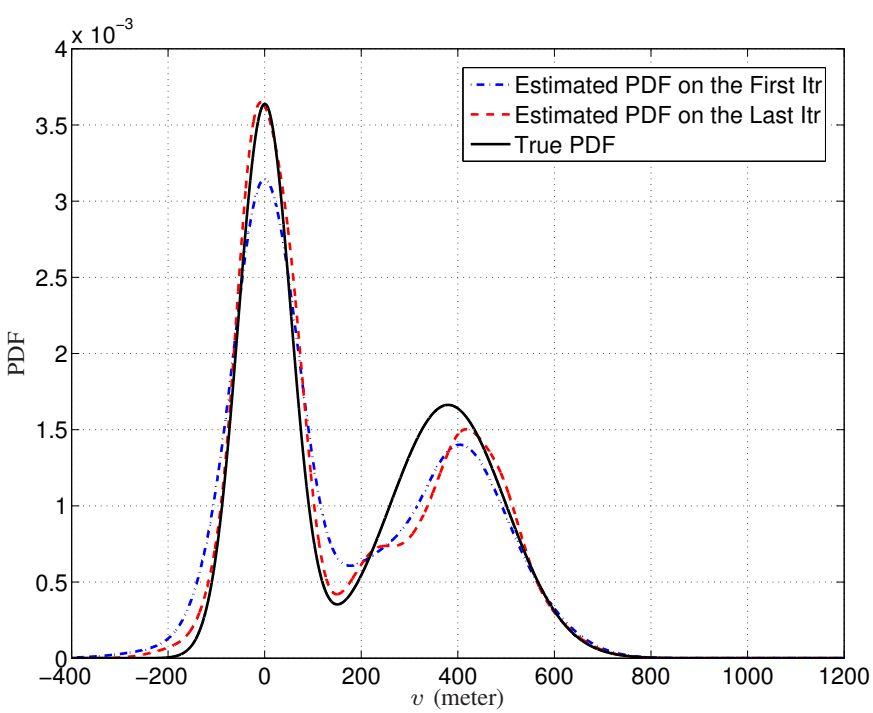

Fig. 6. A comparison among the exact measurement error $\operatorname{PDF} p_{V}(v)$ (black solid line), the PDF estimated on the last iteration of Algorithm 1 (red dashed line) and the PDF estimated on the first iteration of the Algorithm 1 (as same as the one constructed in the robust nonparametric approach [32]) (blue dashdot line) in a particular Monte Carlo trial with $[x=250 \mathrm{~m}, y=250 \mathrm{~m}]^{T}$, $K=20$ samples and $\varepsilon=0.5$.

as follows:

1) For the first two robust estimators developed under the linear regression model, the measurement error $v_{i}(k)$ is most probably generated from the LOS mixture component $\mathcal{N}\left(v ; 0, \sigma_{\mathrm{L}}^{2}\right)$ resulting in $\tilde{v}_{i}(k) \approx 2 h_{i}(\boldsymbol{\theta}) \cdot v_{i}(k)$ in (8) for our simulation scenario. As a result, $\tilde{v}_{i}(k)$, $i=1,2, \ldots, N$ and $k=1,2, \ldots, K$ can be regarded as approximately jointly Gaussian distributed, and it was shown in [29], [30] that they perform nearly optimally under the Gaussian model.

2) Due to the good quality of the initial guess (i.e., the least-squares estimate), the estimated measurement error PDF obtained on the first iteration of Algorithm 1 is already close to the exact PDF and extra iterations can only ameliorate it slightly. This explains why the robust nonparametric estimator and the new proposed one show similar performance as that of the MLE.

Secondly, the new proposed estimator is closest to the MLE and outperforms by far all the other robust competitors when $\varepsilon$ is large. The reasons are as follows:

1) When $\varepsilon$ is large, the PDF of $\tilde{v}_{i}(k)$ starts to deviate from the Gaussian model, leading to deteriorated performance of the robust estimators developed under the linear regression model.

2) The robust M-estimator breaks down at best for a contamination ratio equal to 0.5 [14]. Therefore, it is not surprising to see the drastic performance degradation from $\varepsilon>0.3$ in Fig. 4 .

3) The existing robust semi-parametric estimator [30] performs even worse than the least-squares estimator or the robust M-estimator for some $\varepsilon$ (e.g., $\varepsilon=0.9$ in Fig. 4). The reason may lie in the fact that the transformation function $t(\hat{\tilde{\mathbf{v}}} ; \zeta)$ and the associated search
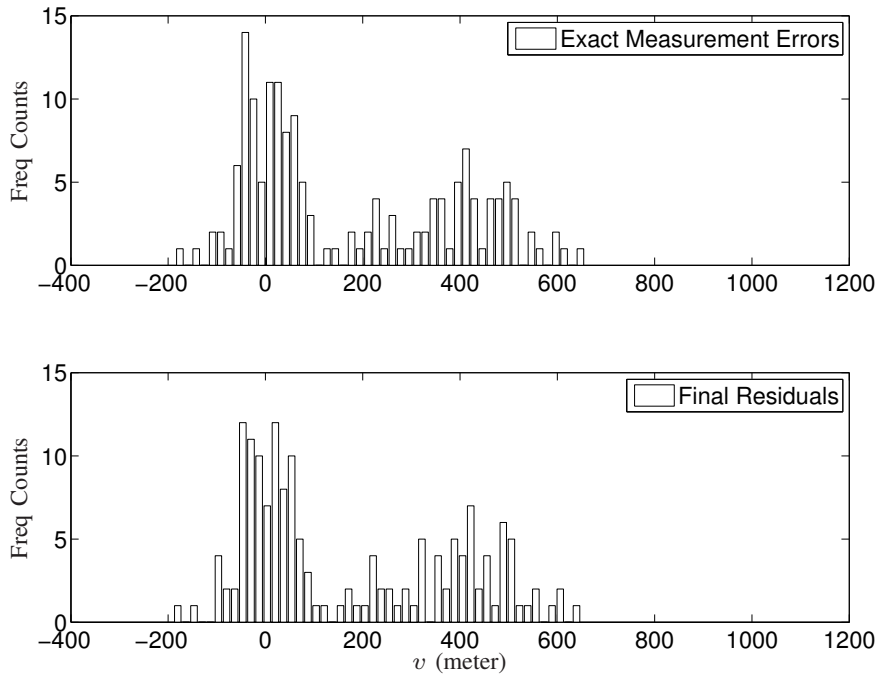

Fig. 7. Histogram of the exact measurement errors (upper) versus the histogram of the residuals extracted on the last iteration of Algorithm 1 (lower) in the same Monte Carlo trial. Note that the sum of frequency counts shown in this figure is equal to the number of measurements, $N K=160$ samples.

interval $\left[\zeta_{L}=0.9, \zeta_{U}=1\right]$ needed in the TKDE are inappropriate for our assumed simulation scenario. ${ }^{5}$

4) Having conquered the drawbacks of the existing semiparametric approach (cf. Section III-A), we harvest improved performance in terms of bias and RMSE in both the robust nonparametric approach [32] and the newly proposed approach.

5) Since the quality of the initial guess (i.e., the leastsquares estimate) degenerates as $\varepsilon$ increases, larger discrepancy has been observed between the exact measurement error PDF $p_{V}(v)$ and its estimate calculated on the first iteration of Algorithm 1. Therefore, the robust nonparametric approach becomes worse as $\varepsilon$ increases. Introducing extra iterations in the newly proposed approach, we successfully reduce the discrepancy and consequently achieve further improved geolocation accuracy.

In the second example, the overall performance of the position estimators in the city center area is examined. We generate 2000 sets of different geographic coordinates $[x, y]^{T}$ uniformly from the city center area, calculate the RMSE and CRLB $_{\text {pos }}$ for each set, and finally average them all. The results are shown in Fig. 5, from which we observe that the performance of the proposed estimator and MLE stays almost unaltered while the performance of the others becomes worse. Here, it is noteworthy that the RMSE curve of the MLE coincides well with the corresponding $\mathrm{CRLB}_{\text {pos }}$ curve in Fig. 4 and Fig. 5.

\section{Measurement Error PDF Estimation via AKDE}

In the previous simulation, we have seen a performance improvement in the newly proposed approach as compared to

${ }^{5}$ The transformation function $t(\tilde{v} ; \zeta)$ and the associated search interval for $\zeta,\left[\zeta_{L}=0.9, \zeta_{U}=1\right]$, were demonstrated to be optimal for the simulation scenario defined in [30] which is different from ours. 
the robust nonparametric approach. As analyzed beforehand, this improvement mainly stems from the extra enhancement in the PDF estimation achieved in the new algorithm. In order to confirm our statement, Fig. 6 shows the PDF estimates obtained on different iterations of Algorithm 1 versus the exact measurement error PDF for a particular Monte Carlo trial in the first example of Section VI-B. Moreover, Fig. 7 shows the histogram of both the exact measurement errors and final residuals (cf. Step 2 of Algorithm 1) of this particular trial. It is worth emphasizing that the enhancement in the PDF estimation is clearly seen in almost every trial, but due to space limitations only one snapshot is shown here.

\section{Estimation Efficiency}

Next, we show the estimation efficiency of the proposed position estimator as a function of the number of measurements. Here, we assume that the MS is located at $[x=$ $250 \mathrm{~m}, y=250 \mathrm{~m}]^{T}, K$ varies from 5 to 75 at an increment of 5 samples and $\varepsilon=0.5$. For each $K, M C=1000$ Monte Carlo trials are carried out to calculate the RMSE, and then the estimation efficiency according to (34) is evaluated. Fig. 8 shows the resulting estimation efficiency versus the number of measurements at each BS. Gathering these results, we summarize the performance of the newly proposed estimator as follows:

- It performs closer to the MLE as $N K$ increases. The estimation efficiency of the MLE is very close to one when $N K \geq 450$ samples.

- It achieves good estimation efficiency for large $N K$. When $N K=100$ samples are available, it attains an efficiency of $\eta \approx 0.8$; When we further increase $N K$ to 300 samples, the efficiency is $\eta \approx 0.9$; Although not shown here, the efficiency will increase very slowly thereafter and attain $\eta \approx 1$ at $N K=1000$ samples.

- It largely deviates from the MLE when $N K$ is small (e.g., $N K \leq 50$ samples), but still outperforms other robust estimators by far.

In addition, we also found that the existing robust semiparametric estimator in [30] does not fulfill the asymptotic efficiency property.

\section{E. Computational Complexity and Speed of Convergence}

One of the most important location-based services is the E-911 service, where the time consumed in estimating the position of a caller in emergency must be restricted to 30 seconds [8]. In order to examine our new approach in this aspect, we calculate the average computational time it requires to give an individual position estimate in the second simulation. The results in Table II show that the average computational time of the new approach is higher than other approaches, but it is still acceptable for the emergency use. Besides, it needs circa 6 iterations on average for Algorithm 1 to converge. ${ }^{6}$

\footnotetext{
${ }^{6}$ All the simulations have been performed under MATLAB ${ }^{T M}$ R2010a environment on a PC equipped with Intel $\left(\right.$ Core $^{\mathrm{TM}} \mathrm{i} 5-760$ processor $(2.80 \mathrm{GHz})$ and 8GB RAM.
}

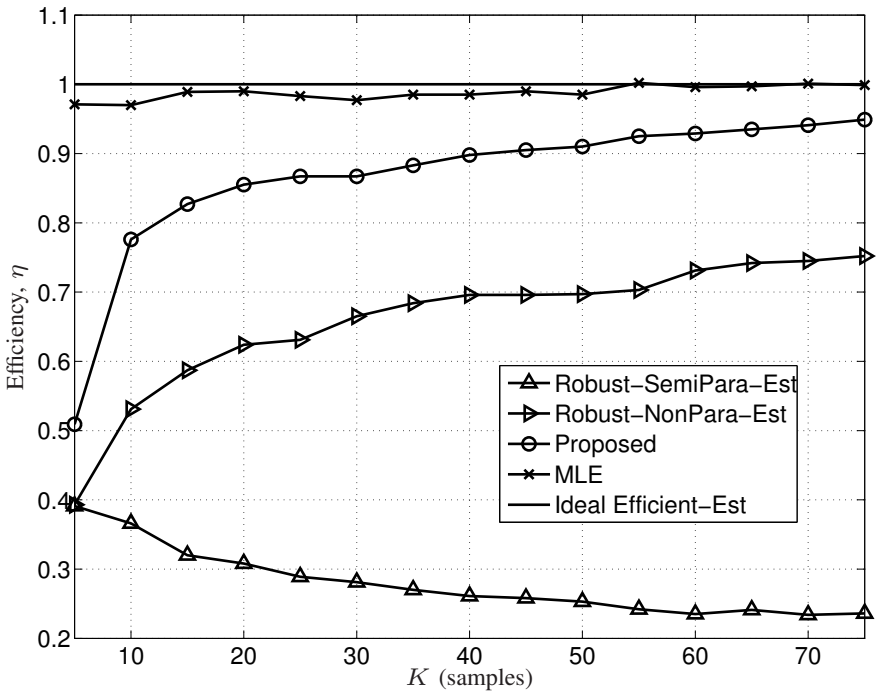

Fig. 8. Estimation efficiency $\eta$ of different position estimators versus the number of measurements collected at each of $N=8 \mathrm{BSs}, K$.

TABLE II

AVERAGE COMPUTATIONAL TIME FOR GENERATING A SINGLE POSITION ESTIMATE IN THE SECOND SIMULATION

\begin{tabular}{l|c}
\hline Name & Computational time \\
\hline Least-squares approach & $<0.0001 \mathrm{sec}$. \\
Robust M-estimation approach & $0.0789 \mathrm{sec}$. \\
Robust semi-parametric approach & $0.6273 \mathrm{sec}$. \\
Robust nonparametric approach & $0.6580 \mathrm{sec}$. \\
Proposed approach & $2.4284 \mathrm{sec}$. \\
E-911 requirement & $<30 \mathrm{sec}$. \\
\hline
\end{tabular}

F. Remark on Simulation Results and Practical Implementation

In the above simulations, we focus on the Gaussian mixture model, although the proposed algorithm is able to work for any other model. The overwhelming reason is that a Gaussian mixture can approximate any other distribution arbitrarily well in, for instance, the $\mathcal{L}_{1}$ norm. Therefore, we find the Gaussian mixture case is the most representative example. For rigor, we have also tested the newly proposed approach in the scenarios where different measurement error distributions (e.g., the twomode Gaussian-Rayleigh and Gaussian-exponential mixture) and simulated BS geometries (e.g., the one utilized in [32]). Similar conclusions can be drawn in these cases.

Here, we use $N=8$ BSs for geolocation. However, in practice, not all of the possible BSs are detectable, implying a smaller $N$ is available. In this case, our algorithm will show moderately degraded performance in the localization (cf. Section VI-B) and PDF approximation (cf. Section VI-C) in the above examples, since less total number of measurements $N K$ are available. However, the key conclusions drawn in Section VI would not change at all. In order to compensate for the performance loss due to the smaller $N$, we can simply collect more samples, $K$, at each BS.

Moreover, we briefly discuss two important aspects concerning practical implementation of our proposed algorithm. The first one is how to predetermine $K$-the number of measurements collected at each BS. It can be determined in 
an offline training phase as follows: First, we test different $K \mathrm{~s}$ for each of the positions in an area of interest and register the resulting geolocation accuracies produced by our proposed algorithm. Next, we choose the smallest $K$ amongst all tested values that satisfies the given requirement(s), e.g., the Federal Communications Commission (FCC) requirement, for the subsequent online localization phase. The second aspect lies in the selection of an initial position estimate. Our preference is the least-squares position estimate. If it is found unreliable, we could resort to the sophisticated strategy presented previously. Besides, we could also integrate the information, if available, from global positioning system (GPS) or assisted-GPS.

\section{G. Remark on Possible Applications}

Before concluding our paper, another important remark is given here. In fact, the route we have followed to design the new robust position estimator in Section III and compute the Cramér-Rao lower bound in Section V are directly applicable to a general measurement model, where $\mathbf{r}$ is a measurement vector, $\boldsymbol{\theta}$ is a deterministic vector parameter to be determined, $\mathbf{h}(\boldsymbol{\theta})$ is an arbitrary nonlinear mapping matrix, and $\mathbf{v}$ is a nonGaussian noise vector of i.i.d. elements with unknown PDF. Many signal processing problems arrive at this generalized measurement model, for instance:

1) Geolocation using a single moving receiver with any type of measurements such as TOA, TDOA, RSS and AOA, e.g., [8, Chap. 17].

2) Source localization and tracking in wireless sensor networks, e.g., [61].

3) Direction-of-arrival (DOA) estimation in non-Gaussian noise, e.g., [62].

4) Amplitude estimation of sinusoidal signals in nonGaussian noise, e.g., [63].

\section{CONCLUSION}

We have developed an iterative algorithm for robust position estimation in harsh line-of-sight/non-line-of-sight environments, where the measurement error is assumed to be completely unknown. We have also presented the best achievable geolocation accuracy in terms of the CRLB in the assumed situation. Simulations have been performed both in the realworld and simulated scenarios. The results show that the proposed position estimator achieves significantly improved performance. Especially when the number of range measurements is large, our new proposed position estimator is very close to the MLE and the geolocation accuracy approaches the best achievable performance. However, the improvement comes at the expense of higher computation as compared to other competitors.

\section{APPENDIX A}

\section{Proof of The Diagonal Property of $\boldsymbol{\mathcal { I }}_{\mathbf{v}}$}

Due to the statistical properties of the vector elements $v_{m}$, $m=1,2, \ldots, N K$, the $\left(m, m^{\prime}\right)$ th entry of the matrix $\mathcal{I}_{\mathbf{v}}$ can be expressed by

$$
\left[\mathcal{I}_{\mathbf{v}}\right]_{m, m^{\prime}}=\mathbb{E}_{p_{\mathbf{v}}(\mathbf{v})}\left\{\frac{\nabla_{v_{m}} p_{V}\left(v_{m}\right) \cdot \nabla_{v_{m^{\prime}}} p_{V}\left(v_{m^{\prime}}\right)}{p_{V}\left(v_{m}\right) \cdot p_{V}\left(v_{m^{\prime}}\right)}\right\} .
$$

If $m=m^{\prime}$, the $m$ th main diagonal element is simplified to

$$
\left[\mathcal{I}_{\mathbf{v}}\right]_{m, m}=\mathcal{I}_{v_{m}}=\mathbb{E}_{p_{V}\left(v_{m}\right)}\left\{\frac{\left[\nabla_{v_{m}} p_{V}\left(v_{m}\right)\right]^{2}}{\left[p_{V}\left(v_{m}\right)\right]^{2}}\right\} .
$$

Since $v_{m}, m=1,2, \ldots, N K$ are identically distributed, we can conclude that

$$
\mathcal{I}_{v}=\mathcal{I}_{v_{1}}=\mathcal{I}_{v_{2}}=\ldots=\mathcal{I}_{v_{N K}} .
$$

if $m \neq m^{\prime},\left[\mathcal{I}_{\mathbf{v}}\right]_{m, m^{\prime}}$ is equal to

$$
\mathbb{E}_{p_{V}\left(v_{m}\right)}\left\{\frac{\nabla_{v_{m}} p_{V}\left(v_{m}\right)}{p_{V}\left(v_{m}\right)}\right\} \cdot \mathbb{E}_{p_{V}\left(v_{m^{\prime}}\right)}\left\{\frac{\nabla_{v_{m^{\prime}}} p_{V}\left(v_{m^{\prime}}\right)}{p_{V}\left(v_{m^{\prime}}\right)}\right\},
$$

since $v_{m}$ and $v_{m^{\prime}}$ are mutually independent. In order to prove the diagonal property of $\mathcal{I}_{\mathbf{v}}$, we recall that for the computation of the CRLB, the following regularity condition

$$
\mathbb{E}_{p(\mathbf{r} \mid \boldsymbol{\theta})}\left\{\nabla_{\boldsymbol{\theta}} \log p(\mathbf{r} \mid \boldsymbol{\theta})\right\}=\mathbf{0}
$$

must hold for any $\boldsymbol{\theta}$. Performing the chain rule [55] upon $\nabla_{\boldsymbol{\theta}} \log p(\mathbf{r} \mid \boldsymbol{\theta})$ in (43), we can easily obtain

$$
\left[\nabla_{\boldsymbol{\theta}} \mathbf{h}(\boldsymbol{\theta})\right] \cdot \mathbb{E}_{p_{\mathbf{v}}(\mathbf{v})}\left\{\frac{\nabla_{\mathbf{v}} p_{\mathbf{v}}(\mathbf{v})}{p_{\mathbf{v}}(\mathbf{v})}\right\}=\mathbf{0}
$$

for any $\boldsymbol{\theta}$, implying that

$$
\mathbb{E}_{p_{V}(v)}\left\{\frac{\nabla_{v} p_{V}(v)}{p_{V}(v)}\right\}=0
$$

because the elements in $\mathbf{v}$ are i.i.d.. As a result, $\left[\mathcal{I}_{\mathbf{v}}\right]_{m, m^{\prime}}=0$ for $m \neq m^{\prime}$ and $\left[\mathcal{I}_{\mathbf{v}}\right]_{m, m^{\prime}}=\mathcal{I}_{v}$ for $m=m^{\prime}$, i.e., $\mathcal{I}_{\mathbf{v}}=$ $\mathcal{I}_{v} \cdot \mathbf{I}_{N K}$ is proven.

\section{REFERENCES}

[1] A. H. Sayed, A. Tarighat, and N. Khajehnouri, "Network-based wireless location: challenges faced in developing techniques for accurate wireless location information," IEEE Signal Process. Mag., vol. 22, no. 4, pp. 24-40, Jul. 2005.

[2] J. J. Caffery and G. L. Stuber, "Overview of radiolocation in CDMA cellular systems," IEEE Commun. Mag., vol. 36, no. 4, pp. 38-45, Apr. 1998.

[3] A. Amar and A. J. Weiss, "Localization of narrowband radio emitters based on Doppler frequency shifts," IEEE Trans. Signal Process., vol. 56, no. 11, pp. 5500-5508, Nov. 2008.

[4] A. J. Weiss, "Direct geolocation of wideband emitters based on delay and Doppler," IEEE Trans. Signal Process., vol. 59, no. 6, pp. 25132521, Jun. 2011.

[5] J. J. Caffery, Wireless Location in CDMA Cellular Radio System. Norwell, MA: Kluwer Academic Publisher, 1999.

[6] F. Gustafsson and F. Gunnarsson, "Mobile positioning using wireless networks: possibilities and fundamental limitations based on available wireless network measurements," IEEE Signal Process. Mag., vol. 22, no. 4, pp. 41-53, Jul. 2005.

[7] S. Gezici, "A survey on wireless position estimation," Wireless Pers. Commun., vol. 44, pp. 263-282, Feb. 2008.

[8] R. Zekavat and R. M. Buehrer, Handbook of Position Location. Hoboken, NJ: John Wiley \& Sons, Inc., 2011.

[9] Y. T. Chan and K. C. Ho, "A simple and efficient estimator for hyperbolic location," IEEE Trans. Signal Process., vol. 42, no. 8, pp. 1905-1915, Aug. 1994.

[10] K. W. Cheung, H. C. So, W. K. Ma, and Y. T. Chan, "Least squares algorithms for time-of-arrival-based mobile location," IEEE Trans. Signal Process., vol. 52, no. 4, pp. 1121-1130, Apr. 2004.

[11] K. W. Cheung and H. C. So, "A multidimensional scaling framework for mobile location using time-of-arrival measurements," IEEE Trans. Signal Process., vol. 53, no. 2, pp. 460-470, Feb. 2005.

[12] H.-W. Wei, Q. Wan, Z.-X. Chen, and S.-F. Ye, "A novel weighted multidimensional scaling analysis for time-of-arrival-based mobile location," IEEE Trans. Signal Process., vol. 56, no. 7, pp. 3018-3022, Jul. 2008. 
[13] H. C. So and F. K. W. Chan, "A generalized subspace approach for mobile positioning with time-of-arrival measurements," IEEE Trans. Signal Process., vol. 55, no. 10, pp. 5103-5107, Oct. 2007.

[14] H. C. So, Y. T. Chan, and F. K. W. Chan, "Closed-form formulae for time-difference-of-arrival estimation," IEEE Trans. Signal Process., vol. 56, no. 6, pp. 2614-2620, Jun. 2008.

[15] Y. T. Chan, W. Y. Tsui, H. C. So, and P. C. Ching, "Time-of-arrival based localization under NLOS conditions," IEEE Trans. Veh. Technol., vol. 55, no. 1, pp. 17-24, Jan. 2006

[16] J. Riba and A. Urruela, "A non-line-of-sight mitigation technique based on ML-detection," in Proc. IEEE Int. Conf. Acoustics, Speech, and Signal Processing (ICASSP), vol. 2, Montreal, Quebec, Canada, May 2004, pp. 153-156

[17] Y. H. Qi, H. Kobayashi, and H. Suda, "Analysis of wireless geolocation in a non-line-of-sight environment," IEEE Trans. Wireless Commun., vol. 5, no. 3, pp. 672-681, Mar. 2006.

[18] X. Wang, Z. X. Wang, and B. O'Dea, "A TOA-based location algorithm reducing the errors due to non-line-of-sight (NLOS) propagation," IEEE Trans. Veh. Technol., vol. 52, no. 1, pp. 112-116, Jan. 2003.

[19] S. Venkatesh and R. M. Buehrer, "A linear programming approach to NLOS error mitigation in sensor networks," in Proc. IEEE Int. Conf. Information Processing in Sensor Networks (IPSN), Nashville, TN, USA, Apr. 2006, pp. 301-308.

[20] H. Y. Chen, G. Wang, Z. Z. Wang, H. C. So, and H. V. Poor, "Nonline-of-sight node localization based on semi-definite programming in wireless sensor networks," IEEE Trans. Wireless Commun., vol. 11, no. 1, pp. 108-116, Jan. 2012.

[21] R. A. Maronna, R. D. Martin, and V. J. Yohai, Robust Statistics: Theory and Methods. Chichester, England: John Wiley \& Sons Ltd., 2006.

[22] P. J. Huber and E. M. Ronchetti, Robust Statistics. Hoboken, NJ: John Wiley \& Sons Ltd., 2009.

[23] Z. Li, W. Trappe, Y. Zhang, and B. Nath, "Robust statistical methods for securing wireless localization in sensor networks," in Proc. IEEE Int. Symp. Information Processing in Sensor Networks (IPSN), Los Angeles, CA, Apr. 2005, pp. 91-98.

[24] R. Casas, A. Marco, J. J. Guerrero, and J. Falcó, "Robust estimator for non-line-of-sight error mitigation in indoor localization," EURASIP J. Appl. Signal Process., vol. 2006, no. 1, pp. 1-8, Jan. 2006.

[25] G. L. Sun and W. Guo, "Bootstrapping M-estimators for reducing errors due to non-line-of-sight (NLOS) propagation," IEEE Commun. Lett., vol. 8, no. 8, pp. 509-510, Aug. 2004.

[26] A. M. Zoubir and D. R. Iskander, Bootstrap Techniques for Signal Processing. Cambridge, U.K.: Cambridge University Press, 2004.

[27] A. M. Zoubir and B. Boashash, "The bootstrap and its application in signal processing," IEEE Signal Process. Mag., vol. 15, no. 1, pp. 56-76, Jan. 1998.

[28] A. M. Zoubir and D. R. Iskander, "Bootstrap methods and applications," IEEE Signal Process. Mag., vol. 24, no. 4, pp. 10-19, Jul. 2007.

[29] U. Hammes, "Robust positioning algorithm for wireless networks," Ph.D. dissertation, Technische Universität Darmstadt, Dec. 2009.

[30] U. Hammes, E. Wolsztynski, and A. M. Zoubir, "Robust tracking and geolocation for wireless networks in NLOS environments," IEEE J. Sel. Topics. Signal Process., vol. 3, no. 5, pp. 889-901, Oct. 2009.

[31] I. Guvenc and C. C. Chong, "A survey on TOA based wireless localization and NLOS mitigation techniques," Commun. Surveys Tuts., vol. 11, no. 3, pp. 107-124, third quarter 2009.

[32] F. Yin and A. M. Zoubir, "Robust positioning in NLOS environments using nonparametric adaptive kernel density estimation," in Proc. IEEE Int. Conf. Acoustics, Speech and Signal Processing (ICASSP), Kyoto, Japan, Mar. 2012, pp. 3517-3520.

[33] B. W. Silverman, Density Estimation: for Statistical and Data Analysis. London: Chapman and Hall, 1986.

[34] J.-F. Liao and B.-S. Chen, "Robust mobile location estimator with NLOS mitigation using interacting multiple model algorithm," IEEE Trans. Wireless Commun., vol. 5, no. 11, pp. 3002-3006, Nov. 2006

[35] C. Fritsche, U. Hammes, A. Klein, and A. M. Zoubir, "Robust mobile terminal tracking in NLOS environments using interacting multiple model algorithm," in Proc. IEEE Int. Conf. Acoustics, Speech and Signal Processing (ICASSP), Taipei, Taiwan, Apr. 2009, pp. 3049-3052.

[36] C. Fritsche and A. Klein, "On the performance of mobile terminal tracking in urban GSM networks using particle filters," in Proc. European Signal Process. Conf. (EUSIPCO), Glasgow, Scotland, Aug. 2009, pp. 1953-1957.

[37] U. Hammes and A. M. Zoubir, "Robust mobile terminal tracking in NLOS environments based on data association," IEEE Trans. Signal Process., vol. 58, no. 11, pp. 5872-5882, Nov. 2010.
[38] — , "Robust MT tracking based on M-estimation and interacting multiple model algorithm," IEEE Trans. Signal Process., vol. 59, no. 7, pp. 3398-3409, Jul. 2011.

[39] J. M. Huerta and J. Vidal, "Mobile tracking using UKF, time measures and LOS-NLOS expert knowledge," in Proc. IEEE. Int. Conf. Acoustics, Speech, and Signal Processing (ICASSP), vol. 4, Philadelphia, PA, USA, Mar. 2005, pp. 901-904.

[40] L. Cong and W. Zhuang, "Nonline-of-sight error mitigation in mobile location," IEEE Trans. Wireless Commun., vol. 4, no. 2, pp. 560-573, Mar. 2005.

[41] P. C. Chen, "A non-line-of-sight error mitigation algorithm in location estimation," in Proc. IEEE Int. Conf. Wireless Commun. Networking (WCNC), vol. 1, New Orleans, LA, Sep. 1999, pp. 316-320.

[42] M. McGuire, K. N. Plataniotis, and A. N. Venetsanopoulos, "Data fusion of power and time measurements for mobile terminal location," IEEE Trans. Mobile Comput., vol. 4, no. 2, pp. 142-153, Mar./Apr. 2005.

[43] U. Hammes, E. Wolsztynski, and A. M. Zoubir, "Transformation-based robust semiparametric estimation," IEEE Signal Process. Letters, vol. 15, pp. 845-848, 2008.

[44] A. M. Zoubir and R. F. Brcich, "Multiuser detection in heavy tailed noise," Digital Signal Process., vol. 12, no. 2-3, pp. 262-273, 2002.

[45] A. Beck, P. Stoica, and J. Li, "Exact and approximate solutions of source localization problems," IEEE Trans. Signal Process., vol. 56, no. 5, pp. 1770-1778, May 2008.

[46] C. T. Kelley, Solving nonlinear equations with Newton's method, ser. Fundamentals of Algorithms. Philadelphia, PA: Society for Industrial and Applied Mathematics (SIAM), 2003.

[47] R. Fletcher, Practical methods of optimization (2nd ed.). Chichester, England: John Wiley \& Sons Ltd., 1987.

[48] J. A. Bilmes, "A gentle tutorial on the EM algorithm and its application to parameter estimation for Gaussian mixture and hidden Markov models," University of California, Berkeley, Berkeley, CA, USA, Tech. Rep., 1998.

[49] S. Kay, Fundamentals of Statistical Signal Processing: Estimation Theory. Englewood Cliffs, NJ: Prentice-Hall, Inc., 1993.

[50] Y. Bar-Shalom, X. R. Li, and T. Kirubarajan, Estimation with Applications to Tracking and Navigation. New York, NY: John Wiley \& Sons, Inc., 2001.

[51] J. Nocedal and S. J. Wright, Numerical optimization. New York, NY: Springer-Verlag New York, Inc., 1999.

[52] I. S. Abramson, "On bandwidth variation in kernel estimates-a square root law," Ann. Statist., vol. 10, no. 4, pp. 1217-1223, 1982.

[53] F. Gustafsson, Statistical Sensor Fusion. Lund, Sweden: Studentlitteratur, 2012.

[54] C. P. Robert and G. Casella, Monte Carlo Statistical Methods. New York, NY: Springer-Verlag, 1999.

[55] E. L. Lehmann and G. Casella, Theory of point estimation. New York, NY: Springer-Verlag, Inc., 1998

[56] H. L. Van Trees, Optimum Array Processing. Part IV of Detection, Estimation, and Modulation Theory. John Wiley \& Sons, Inc., New York, USA, 2002.

[57] G. Hendeby, "Performance and implementation aspects of nonlinear filtering," Ph.D. dissertation, Linköping University, Linköping, Sweden, Feb. 2008 .

[58] K. C. Ho, "Bias reduction for an explicit solution of source localization using TDOA," IEEE Trans. Signal Process., vol. 60, no. 5, pp. 21012114, May 2012.

[59] N. Levanon, "Lowest GDOP in 2-D scenarios," Proc. IEE Radar, Sonar and Navigation, vol. 147, no. 3, pp. 149-155, Jun. 2000.

[60] C. Fritsche, "Statistical data fusion for hybrid localization of mobile terminals," Ph.D. dissertation, Technische Universität Darmstadt, Mar 2011.

[61] Y. Liu, Y.-H. Hu, and Q. Pan, "Distributed, robust acoustic source localization in a wireless sensor network," IEEE Trans. Signal Process., vol. 60 , no. 8, pp. 4350-4359, Aug. 2012.

[62] R. J. Kozick and B. M. Sadler, "Maximum-likelihood array processing in non-Gaussian noise with Gaussian mixtures," IEEE Trans. Signal Process., vol. 48, no. 12, pp. 3520-3535, Dec. 2000.

[63] P. Stoica, H.-B. Li, and J. Li, "Amplitude estimation of sinusoidal signals: survey, new results, and an application," IEEE Trans. Signal Process., vol. 48, no. 2, pp. 338-352, Feb. 2000. 


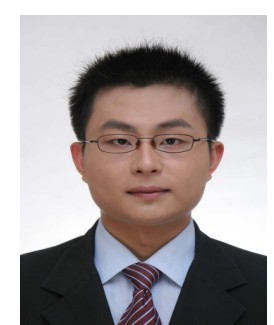

Feng Yin received the B.Sc. degree from Shanghai Jiao Tong University, China in 2008, and the M.Sc. degree from Technische Universität Darmstadt (TUD), Darmstadt, Germany in 2011.

$\mathrm{He}$ is currently working towards the Ph.D. degree in the Signal Processing Group (SPG) at Technische Universität Darmstadt. His research interests include optimal waveform design, robust estimation theory with applications to wireless geolocation, tracking and navigation.

Carsten Fritsche received the Dipl.-Ing. degree in 2005 and the Dr.-Ing. degree in 2011 both in electrical engineering and information technology from Technische Universität Darmstadt, Germany. In 2011 he joined the Division of Automatic Control at Linköping University, Sweden, as a postdoctoral research fellow. Since spring 2012, he has been with IFEN GmbH, Poing, Germany, working as a systems engineer on satellite navigation related projects. His main research interest lies in the area of statistical signal processing, machine learning and sensor fusion with applications to wireless positioning and satellite navigation.

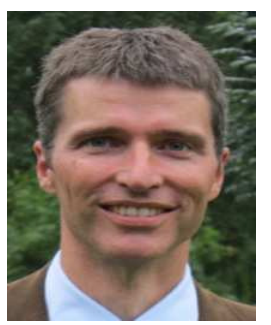

Fredrik Gustafsson is professor in Sensor Informatics at Department of Electrical Engineering, Linköping University, since 2005. He received the M.Sc. degree in electrical engineering 1988 and the Ph.D. degree in Automatic Control, 1992, both from Linköping University. During 1992-1999 he held various positions in automatic control, and 19992005 he had a professorship in Communication Systems. His research interests are in stochastic signal processing, adaptive filtering and change detection, with applications to communication, vehicular, airborne, and audio systems. He is a co-founder of the companies NIRA Dynamics (automotive safety systems), Softube (audio effects) and SenionLab (indoor positioning systems).

He was an associate editor for IEEE Transactions on Signal Processing 2000-2006 and is currently associate editor for IEEE Transactions on Aerospace and Electronic Systems and EURASIP Journal on Applied Signal Processing. He was awarded the Arnberg prize by the Royal Swedish Academy of Science (KVA) 2004, elected member of the Royal Academy of Engineering Sciences (IVA) 2007, elevated to IEEE Fellow 2011 and awarded the Harry Rowe Mimno Award 2011 for the tutorial "Particle Filter Theory and Practice with Positioning Applications", which was published in the AESS Magazine in July 2010.

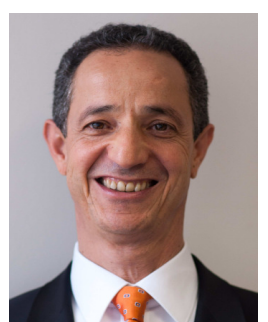

Abdelhak M. Zoubir is a Fellow of the IEEE and IEEE Distinguished Lecturer (Class 2010-2011). He received his Dr.-Ing. from Ruhr- Universität Bochum, Germany in 1992. He was with Queensland University of Technology, Australia from 1992-1998 where he was Associate Professor. In 1999, he joined Curtin University of Technology, Australia as a Professor of Telecommunications and was Interim Head of the School of Electrical \& Computer Engineering from 2001 until 2003. In 2003, he moved to Technische Universität Darmstadt, Germany as Professor of Signal Processing and Head of the Signal Processing Group. His research interest lies in statistical methods for signal processing with emphasis on bootstrap techniques, robust detection and estimation and array processing applied to telecommunications, radar, sonar, automotive monitoring and safety, and biomedicine. He published over 300 journal and conference papers on these areas. Professor Zoubir was the Technical Chair of the 11th IEEE Workshop on Statistical Signal Processing (SSP 2001), General Co-Chair of the 3rd IEEE International Symposium on Signal Processing \& Information Technology (ISSPIT 2003) and of the 5th IEEE Workshop on Sensor Array and Multi-channel Signal Processing (SAM 2008). He is the General CoChair of the 14th International Workshop on Signal Processing Advances in Wireless Communications (SPAWC 2013) to be held in Darmstadt, Germany, the General Co-Chair of the 21st European Signal Processing Conference (EUSIPCO 2013) to be held in Marrakech, Morocco, and the Technical CoChair of ICASSP-14 to be held in Florence, Italy. Dr. Zoubir was an Associate Editor of the IEEE Transactions on Signal Processing (1999-2005), a Member of the Senior Editorial Board of the IEEE Journal on Selected Topics in Signal Processing (2009-2011) and he currently serves on the Editorial Boards of the EURASIP journals Signal Processing and the Journal on Advances in Signal Processing (JASP). He currently serves a three-year term as Editor-In-Chief of the IEEE Signal Processing Magazine (2012-2014). Dr. Zoubir was Past Chair (2012), Chair (2010-2011), Vice-Chair (2008-2009) and Member (20022007) of the IEEE SPS Technical Committee Signal Processing Theory and Methods (SPTM). He was a Member of the IEEE SPS Technical Committee Sensor Array and Multi-channel Signal Processing (SAM) from 2007 until 2012. He also serves on the Board of Directors of the European Association of Signal Processing (EURASIP). 